\title{
Uma revisão dos princípios da conversão fotovoltaica de energia
}

\author{
An overview of photovoltaic energy conversion principles
}

\author{
Ariane A. Lima ${ }^{1}$, Natalia Pereira Menezes ${ }^{1}$, Sthefany Santos ${ }^{1}$, Byanca Amorim ${ }^{1}$, Fabiano Thomazi ${ }^{1}$, \\ Fernando Zanella ${ }^{1}$, Armando Heilmann ${ }^{1}$, E. Burkarter ${ }^{2}$, Cesar A. Dartora*10 \\ ${ }^{1}$ Universidade Federal do Paraná, Departamento de Engenharia Elétrica, Curitiba, PR, Brasil \\ ${ }^{2}$ Instituto Federal do Paraná, Curitiba, PR, Brasil
}

Recebido em Julho 30 de 2019. Revisado em Agosto 27 de 2019. Aceito em Agosto 28 de 2019

\begin{abstract}
A conversão fotovoltaica de energia é uma área de pesquisa muito ativa devido à necessidade de ampliar a participação das fontes de energia renováveis na matriz energética global. Os esforços de pesquisa vão desde a modelagem de dispositivos fotovoltaicos a partir de princípios físicos fundamentais ou fenomenologias, passando pelo desenvolvimento e caracterização de novos materiais e dispositivos aplicáveis na conversão fotovoltaica e indo até os estudos de impacto ambiental e aspectos econômicos. No presente trabalho, utilizando-se do modelo de sistema de dois níveis acoplado a um campo de fótons, apresentam-se os princípios físicos da conversão fotovoltaica de energia. Com isso, além de servir de rápido guia para os pesquisadores e estudantes no campo da conversão fotovoltaica, o problema spin-bóson é utilizado de forma pedagógica para servir como um exercício contextualizado de mecânica quântica e física dos semicondutores.
\end{abstract}

Palavras-chave: célula solar, conversão fotovoltaica, fóton, semicondutores, polímeros fotoativos.

Photovoltaic energy conversion is an extraordinarily active research field, mainly due to the need for increasing the participation of renewable energy sources in the world energy matrix. Research efforts range from the photovoltaic devices modelling from first principles or using phenomenology and the development and characterization of novel photovoltaic materials and devices to environmental and economical aspects of photovoltaic energy. The present work presents the physical aspects of photovoltaic energy conversion, using in a pedagogical way a two system level coupled to a photon field as a toy model to discuss the main ideas, serving as a quick guide for researchers and students in the field.

Keywords: solar cell, photovoltaic conversion, photon, semiconductors, photoactive polymers.

\section{Introdução}

O efeito fotovoltaico foi descoberto em 1839 pelo físico francês Edmond Becquerel, (pai de Henri Becquerel, famoso pelos estudos em radioatividade), utilizando uma célula eletroquímica para gerar uma diferença de potencial entre dois eletrodos, quando o dispositivo era submetido à luz [1]. Os primeiros painéis solares foram desenvolvidos por Charles Fritts nos anos 1880 [2] e instalados em um telhado na cidade de Nova Iorque, mas somente a partir dos anos 1940, através da patente [3] e dos trabalhos de Russel Ohl, os painéis solares utilizando junções p-n de silício tornaram-se viáveis para aplicações práticas. Eficiências de conversão maiores que $5 \%$ foram conseguidas a partir da década de 1950, o que foi um salto fundamental para a viabilização das comunicações via satélite.

Na essência, o efeito fotovoltaico corresponde à geração de uma diferença de potencial elétrico entre dois terminais de uma estrutura, usualmente uma junção p-n. No

*Endereço de correspondência: cadartora@eletrica.ufpr.br dispositivo sob iluminação o efeito fotoelétrico interno é capaz de gerar um par elétron-buraco pela absorção de um fóton [4].

$\mathrm{Na}$ atualidade a radiação solar é uma das mais importantes fontes de energia renovável [5-10], sobretudo na região espectral da luz infravermelha e visível, podendo ser convertida diretamente em calor ou em energia elétrica, através de materiais e dispositivos apropriados. Um dispositivo capaz de converte a luz diretamente em energia elétrica, através da produção de uma tensão e uma corrente elétrica sob iluminação, é denominado célula fotovoltaica. Tipicamente, um ponto qualquer na superfície do nosso planeta recebe do Sol uma densidade de potência de aproximadamente $1000 \mathrm{~W} / \mathrm{m}^{2}$, nos momentos de maior iluminação. Essa quantidade varia em função da estação do ano, hora do dia e localização geográfica (quanto maior a latitude menor a incidência de radiação solar). Algumas aplicações tecnológicas atuais são fundamentalmente dependentes da conversão fotovoltaica de energia para operar, como por exemplo, a fonte primária de energia em satélites, naves espaciais, antenas 
repetidoras de sinal e regiões remotas do planeta sem acesso às redes de energia elétrica convencionais.

Desse modo, a conversão fotovoltaica de energia é uma área de pesquisa muito ativa e relevante, atraindo pesquisadores e estudantes de pós-graduação em nível de mestrado e doutorado em todo o mundo [11-13]. As pesquisas na área podem ser classificadas dentre os seguintes tópicos principais: i) modelagem de dispositivos fotovoltaicos a partir de principios físicos fundamentais ou fenomenologias [14-22]; ii) desenvolvimento e caracterização de novos materiais aplicáveis na conversão fotovoltaica [23-32]; iii) desenvolvimento e caracterização de novos dispositivos e/ou técnicas de fabricação com base nos materiais já disponíveis [33-43] e iv) estudos de impacto ambiental e aspectos econômicos [44-47].

No presente trabalho objetiva-se apresentar os princípios físicos fundamentais da conversão fotovoltaica de energia, abordando o primeiro dos itens mencionados acima. Para tanto é utilizado na descrição do fenômeno de absorção de fótons um sistema de dois níveis de energia acoplado a um campo de fótons, na forma mais simples do hamiltoniano de Jaynes-Cummings [48-50]. Com isso, além de servir de rápido guia para os pesquisadores e estudantes no campo da conversão fotovoltaica, o problema spin-bóson é utilizado de forma pedagógica para servir como um exercício contextualizado de mecânica quântica e física dos semicondutores.

O conteúdo do presente trabalho está organizado da seguinte maneira: na Seção 2 uma revisão detalhada dos príncípios físicos fundamentais associados à conversão fotovoltaica. Um modelo simples, baseado no hamiltoniano de spin-fóton, será discutido. A Seção 3 detalha a obtenção do coeficiente de absorção luminosa nos meios materiais a partir da regra de ouro de Fermi. O problema dos semicondutores de gap direto e indireto é também discutido. Na Seção 4, são discutidos os estados localizados e a geração de éxcitons, um importante tópico em materiais fotoativos e dispositivos fotovoltaicos. O fenômeno da fotocondutividade é apresentado de forma simplificada na Seção 5, enquanto o modelo equivalente e os principais parâmetros de caracterização de uma célula solar são apresentados na Seção 6. A Seção 7 apresenta um panorama geral dos tipos de células solares e materiais utilizados na sua construção. Finalmente, na Seção 8 são apresentadas as conclusões gerais deste trabalho.

\section{Princípios físicos da conversão fotovoltaica}

A descrição dos fenômenos fotovoltaicos requer a adequada compreensão da interação da luz com a matéria, cuja natureza é inerentemente quântica, aliada a um modelo físico dos meios materiais considerados. Na descrição semi-clássica de um dispositivo fotovoltaico são ainda necessárias as equações de Maxwell do eletromagnetismo. Os materiais utilizados na conversão fotovoltaica são tipicamente semicondutores, cuja estrutura de bandas de energia é obtida mediante a aplicação das leis da mecânica quântica. Nesta Seção iremos discutir em de- talhes os principais ingredientes da teoria da conversão fotovoltaica.

\subsection{Fótons e a interação da luz com a matéria}

Do ponto de vista clássico, a luz é um fenômeno eletromagnético ondulatório descrito pelas equações de Maxwell clássicas. Entretanto, não é possível compreender inteiramente a interação da luz com a matéria através dessa descrição ondulatória, sendo necessário introduzir o conceito de fóton, que representa o quantum de energia da radiação eletromagnética, evidenciando dessa forma o aspecto corpuscular da luz. A energia de um fóton está associada diretamente à frequência da radiação eletromagnética através da relação de Planck $[51,52]$ :

$$
E_{f}=\hbar \omega
$$

onde $\hbar=1,054 \times 10^{-34}$ J.s é a constante de Planck e $\omega$ é a frequência angular da luz, medida em rad/s no SI. O fóton transporta ainda momento linear $\mathbf{p}$, cuja expressão é dada abaixo:

$$
\mathbf{p}=\hbar \mathbf{k}
$$

onde $\mathbf{k}$ é o vetor de onda, medido em $\mathrm{rad} / \mathrm{m}$, associado ao comprimento de onda $\lambda$ pela expressão $|\mathbf{k}|=k=2 \pi / \lambda=$ $\omega / c$, onde $c$ é a velocidade da luz. O fóton também carrega momento angular, possuindo $\operatorname{spin} s=1$, mas que não nos interessa por ora. Do ponto de vista corpuscular, a interação da luz com a matéria pode ser descrita como a colisão do fóton com as partículas elementares que compõem a matéria, onde o fóton pode ser inteiramente absorvido, emitido ou parcialmente espalhado, desde que as leis de conservação de energia, momento linear, momento angular e carga elétrica sejam satisfeitas.

Tipicamente não observamos o aspecto corpuscular da luz devido à granulosidade da radiação em frequências usualmente empregadas em comunicações, por exemplo. Um único fóton de micro-ondas, cujo espectro situa-se entre $300 \mathrm{MHz}$ e $300 \mathrm{GHz}$, tem energia entre $10^{-6} \mathrm{eV}$ e $10^{-3} \mathrm{eV}$. Dessa forma, uma fonte de RF emitindo uma potência de $10 \mathrm{~mW}$ na faixa de $2,4 \mathrm{GHz}$ (empregada em redes $\mathrm{WiFi}$, por exemplo) emite $6,3 \times 10^{21}$ fótons por segundo, e a interação da luz com a matéria se dá via processos envolvendo muitos fótons, cujo comportamento estatístico é muito bem descrito quantitativamente pelas equações de Maxwell clássicas. Apenas a título de comparação, a cor vermelha no espectro de luz visível tem comprimento de onda de $632 \mathrm{~nm}$ e a energia associada ao fóton vale aproximadamente $2 \mathrm{eV}$. Embora um laser vermelho de $10 \mathrm{~mW}$ emita $3 \times 10^{16}$ fótons por segundo, ainda podendo ter uma descrição clássica satisfatória para a propagação, a energia de um único fóton é várias ordens de grandez maior do que um fóton de micro-ondas, sendo capaz de produzir transições eletrônicas entre as diversas bandas de energia nos materiais semicondutores.

A Tabela 1 mostra as energias típicas dos fótons associados a cada faixa do espectro eletromagnético, para fins de comparação. A divisão das faixas foi adaptada a partir de [52] (ver a página 58 daquela referência). O 
Tabela 1: Espectro eletromagnético e energia do fóton associado (Adaptado da Ref. [52]).

\begin{tabular}{lcc}
\hline Região Espectral & $\lambda$ & $E_{f}$ \\
\hline Radiofrequência (RF) & $\lambda<1 \mathrm{~mm}$ & $E_{f}<1 \mathrm{meV}$ \\
Infravermelho (IR) & $1 \mathrm{~mm}>\lambda>700 \mathrm{~nm}$ & $1 \mathrm{meV}<E_{f}<1,78 \mathrm{eV}$ \\
Visível & $700 \mathrm{~nm}>\lambda>400 \mathrm{~nm}$ & $1,78 \mathrm{eV}<E_{f}<3,1 \mathrm{eV}$ \\
Ultravioleta (UV) & $400 \mathrm{~nm}>\lambda>1 \mathrm{~nm}$ & $3,1 \mathrm{eV}<E_{f}<1,25 \mathrm{keV}$ \\
Raios X & $1 \mathrm{~nm}>\lambda>5 \mathrm{pm}$ & $1,25 \mathrm{keV}<E_{f}<250 \mathrm{keV}$ \\
Raios $\gamma$ & $\lambda<5 \mathrm{pm}$ & $E_{f}>250 \mathrm{keV}$ \\
\hline
\end{tabular}

espectro de luz visível está entre 400nm e 700nm. Raios $\mathrm{X}$ e raios $\gamma$ apresentam energias acima de $1 \mathrm{keV}$.

Toda a matéria ordinária é formada de átomos que isoladamente possuem níveis de energia discretos, determinados mediante a solução da equação de Schrödinger. O estado fundamental de um átomo é aquele de menor energia possível. Por exemplo, o hidrogênio é o átomo mais simples e tem estado fundamental $1 s^{1}$. É possível excitar um átomo, colocando-o em um nível energético maior do que o seu estado fundamental, através da irradiação por luz, com a consequente absorção de fótons, desde que esses possuam energia suficiente para a transição entre os níveis de energia, e que certas regras de seleção sejam satisfeitas. No caso do hidrogênio, a transição mais provável pela absorção de um fóton levao elétron do nível $1 s^{1}$ para o nível $2 p^{1}$, conservando energia e momento angular no processo. Os níveis de energia eletrônicos em um átomo podem ser divididos em: i) níveis de caroço, para aqueles elétrons mais internos e próximos ao núcleo, que portanto ficam mais fortemente ligados, e ii) níveis de valência, que correspondem aos elétrons mais externos. A ligação química é tem sua origem essencialmente na interação entre orbitais de valência dos átomos. Aqueles elétrons mais fortemente ligados somente podem ser excitados para níveis de maior energia por intermédio de fótons de raios X ou do alto UV. A radiação $\gamma$ por sua vez, é capaz de produzir transições de níveis energéticos no interior do próprio núcleo, formado pelos prótons e nêutrons. A partir da faixa do ultravioleta, a radiação é considerada ionizante, pelo fato de que um único fóton possui energia suficiente para arrancar um elétron do átomo, deixando o elétron livre e o átomo ionizado. Por exemplo, o hidrogênio possui um espectro de estados ligados cuja energia é dada aproximadamente por $E_{n}=-13,6 / n^{2}$ $\mathrm{eV}$, onde $n=1,2,3 \ldots$ é o número quântico principal. Para ionizar o hidrogênio em seu estado fundamental $1 s^{1}$, é necessário fazer a transição de $n=1$ para $n \rightarrow \infty$, correspondendo a uma energia do fóton de $13,6 \mathrm{eV}$, que está dentro da faixa do UV.

A matéria em estado sólido é um arranjo estável de átomos capaz de minimizar a energia de todo o conjunto de átomos através da interação entre os orbitais de valência dos mesmos. É sabido, da mecânica quântica, que um interação geralmente produz desdobramentos dos níveis de energia discretos do átomo individual, eliminando degenerescências. No limite de grande número de átomos, os desdobramentos dos níveis atômicos produzem bandas contínuas de níveis de energia permitidos, e entre essas bandas, regiões proibidas, conhecidas como gaps de energia. A estrutura de bandas de um material depende essencialmente dos tipos de átomos que o formam, do número de elétrons de valência e da forma de seus orbitais, que em última análise irá definir as simetrias da rede cristalina resultante. Enquanto um material bom condutor, geralmente metálico, possui sua banda de valência apenas parcialmente preenchida, havendo níveis de energia disponíveis e não preenchidos sem separação por um gap, semicondutores e isolantes possuem um gap de energia entre uma banda totalmente preenchida, denomindada usualmente banda de valência (BV), e uma banda de energia totalmente vazia, denominada banda de condução (BC). A diferença entre isolantes e semicondutores está essencialmente no valor do gap de energia. Enquanto os semicondutores têm gap tipicamente menor que $3 \mathrm{eV}$ os materiais isolantes têm gap maior que $5 \mathrm{eV}$. É possível promover elétrons da banda de valência para a banda de condução de um material através de absorção de fótons. $\mathrm{Na}$ banda de condução os elétrons são relativamente livres para se mover, aumentando assim a condutividade do material, ao passo que deixam uma vacância na banda de valência, denominada buraco, que comporta-se efetivamente como uma carga positiva. Uma vez que a radiação solar tem espectro predominante no visível e infravermelho, sendo fortemente atenuado na região do UV e acima, os materiais empregados na construção de dispositivos fotoativos devem ser sensíveis ao espectro visível e abaixo. Geralmente, materiais semicondutores com gap de energia na faixa entre $0,5 \mathrm{eV}$ e 2 $\mathrm{eV}$, como o silício, o germânio e combinações de elementos como o arseneto de gálio, além de alguns materiais poliméricos orgânicos, apresentam boas características para aplicações fotovoltaicas.

\subsection{Modelo de Jaynes-Cummings da interação da luz com a matéria}

Para compreender um pouco melhor os processos de interação da radiação com a matéria é interessante considerar um modelo simplificado constituído de um sistema de dois níveis de energia $\pm \varepsilon_{0} / 2$, cuja diferença vale exatamente $\varepsilon_{0}$, na presença de radiação de uma única frequência $\omega$. Nesse modelo de brinquedo (em inglês, toy model) o estado de menor energia corresponderia ao elétron na banda de valência, enquanto que no estado de maior energia o elétron é promovido para uma banda de condução, deixando um buraco (ausência do elétron) no estado de menor energia. Por uma questão de simplicidade, mas sem perder a essência do problema, a polarização do fóton será desconsiderada aqui. Tal cenário simplificado é análogo ao problema do spin $1 / 2$ 
na presença de um campo magnético variante no tempo, podendo ser modelado através da utilização das matrizes de Pauli [52]. No formalismo da segunda quantização para o campo eletromagnético, o hamiltoniano do sistema toma a forma mais elementar possível do modelo de Jaynes-Cummings e é dado abaixo [48-50]:

$$
\hat{H}=\frac{\varepsilon_{0}}{2} \sigma_{z}+\hbar \omega \hat{a}^{\dagger} \hat{a}+\gamma \sigma^{+} \hat{a}+\gamma^{*} \sigma^{-} \hat{a}^{\dagger},
$$

onde $\gamma$ é uma constante de acoplamento entre fótons e o sistema de dois níveis, â é um operador bosônico de aniquilação de fóton, $\hat{a}^{\dagger}$ é um operador bosônico de criação de fóton, $\sigma_{z}, \sigma^{+}=\left(\sigma_{x}+i \sigma_{y}\right) / 2$ e $\sigma^{-}=\left(\sigma_{x}-\right.$ $\left.i \sigma_{y}\right) / 2$ são matrizes de Pauli,

$\sigma_{z}=\left(\begin{array}{cc}1 & 0 \\ 0 & -1\end{array}\right), \sigma^{+}=\left(\begin{array}{ll}0 & 1 \\ 0 & 0\end{array}\right), \sigma^{-}=\left(\begin{array}{ll}0 & 0 \\ 1 & 0\end{array}\right)$,

satisfazendo a seguinte álgebra:

$$
\begin{aligned}
{\left[\sigma^{+}, \sigma^{-}\right] } & =\sigma_{z}, \\
{\left[\sigma_{z}, \sigma^{+}\right] } & =2 \sigma_{z}, \\
{\left[\sigma_{z}, \sigma^{-}\right] } & =-2 \sigma_{z},
\end{aligned}
$$

onde $[A, B]=A B-B A$ é o comutador, atuando em um espaço de dois níveis de pseudo-spin, $|\uparrow\rangle=\left(\begin{array}{l}1 \\ 0\end{array}\right)$ e $|\downarrow\rangle=\left(\begin{array}{c}0 \\ 1\end{array}\right)$, que correspondem aos estados de energia $\varepsilon_{0} / 2 \mathrm{e}-\varepsilon_{0} / 2$, sendo autoestados da matriz $\sigma_{z}$ com autovalores +1 e -1 , respectivamente. Fica claro também que $|\downarrow\rangle$ é o estado fundamental, enquanto que $|\uparrow\rangle$ é o estado excitado desse sistema. Os operadores bosônicos $\hat{a}$ e $\hat{a}^{\dagger}$ combinam-se para formar o operador de número de fótons $\hat{n}=\hat{a}^{\dagger} \hat{a}$ e satisfazem a álgebra a seguir:

$$
\left[\hat{a}^{\dagger}, \hat{a}^{\dagger}\right]=[\hat{a}, \hat{a}]=0,\left[\hat{a}, \hat{a}^{\dagger}\right]=1 .
$$

No espaço de número de fótons o estado físico com $n$ fótons é denotado simplesmente por $|n\rangle$, sendo autoestado do operador $\hat{n}$, de tal forma que:

$$
\begin{aligned}
\hat{n}|n\rangle & =n|n\rangle, \\
\hat{a}|n\rangle & =\sqrt{n}|n-1\rangle, \\
\hat{a}^{\dagger}|n\rangle & =\sqrt{n+1}|n+1\rangle,
\end{aligned}
$$

sendo $n=0,1,2,3 \ldots$ um número inteiro. Existe, portanto, um estado denominado de vácuo da radiação, $\operatorname{com} n=0$, que é totalmente destruído pelo operador $\hat{a}$, ou seja, $\hat{a}|0\rangle=0$.

Naturalmente, o estado quântico do sistema físico constituído do pseudo-spin mais fótons da radiação pode ser dado como um produto tensorial entre o estado físico do sistema de dois níveis e o estado quântico da radiação, sendo denotado por $|\uparrow, n\rangle$ ou $\left|\downarrow, n^{\prime}\right\rangle$ ou qualquer combinação linear de estados dessa forma. É importante observar que o espaço de número de fótons tem dimensão infinita, e o sistema pode estar em algum estado com número de fótons indefinido (combinação linear de estados de número de fótons distintos).

Voltanto ao hamiltoniano dado em (3), o primeiro termo representa os dois possíveis estados energéticos do sistema $\pm \varepsilon_{0} / 2$, cuja diferença vale $\varepsilon_{0}$, o segundo termo $\hbar \omega \hat{n}$ é o operador que se refere ao número de fótons presentes no sistema. Os dois termos finais são responsáveis pela interação entre o sistema de dois níveis e os fótons. O termo $\gamma \sigma^{+} \hat{a}$ age sobre um estado $|\downarrow, n\rangle$, produzindo um novo estado $|\uparrow, n-1\rangle$, significando que o sistema de dois níveis é levado do estado fundamental para o estado excitado absorvendo um fóton. Esse operador produz resultado nulo se o sistema já estiver em seu estado excitado $|\uparrow, n\rangle$. Por outro lado, o termo $\gamma^{*} \sigma^{-} \hat{a}^{\dagger}$ é o hermitiano conjugado ao anterior, e produz o efeito inverso, fazendo com que o sistema passe do estado excitado $|\uparrow, n\rangle$ para o estado fundamental $|\downarrow, n+1\rangle$ com a consequente emissão de um fóton. Observe ainda que a ação de $\hat{a}$ produz um fator de proporcionalidade $\sqrt{n}$, significando que somente é possível a absorção de fótons se $n>0$, ou seja, é necessário que existam fótons presentes no sistema para que ocorra absorção. Por outro lado, a ação de $\hat{a}^{\dagger}$ resulta em um fator $\sqrt{n+1}$, significando que mesmo para $n=0$ pode haver emissão de fótons, desde que o sistema de dois níveis esteja num estado excitado de energia. Esse fenômeno que permite a emissão de fótons mesmo na ausência de radiação é denominado emissão espontânea. Por outro lado, havendo fótons presentes no sistema, a probabilidade de ocorrência de emissão aumentará com o número, o que é denominado de emissão estimulada de radiação e é o princípio de funcionamento de um dispositivo laser. Fótons emitidos através da emissão estimulada estarão em fase com a radiação presente, permitindo uma radiação de grande intensidade e altamente coerente em fase.

A Figura 1 ilustra de modo simplificado os processos de absorção, emissão espontânea e emissão estimulada para esse sistema de dois níveis.

Nesse ponto, deixamos para o leitor um interessante exercício de mecânica quântica, que é a obtenção das equações de movimento para os operadores $\sigma_{z}, \sigma^{+}, \sigma^{-}, \hat{a}, \hat{a}^{\dagger}$ e $\hat{n}$, utilizando a equação de Heisenberg para um operador

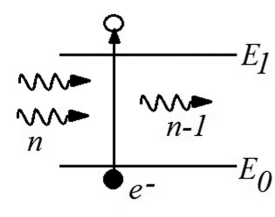

(a)

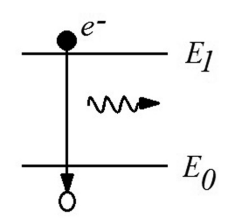

(b)

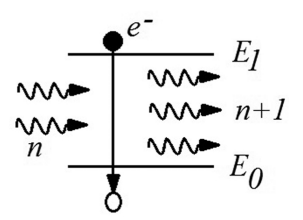

(c)
Figura 1: Interação da radiação com um sistema de dois níveis de energia: a) Absorção, b) Emissão Espontânea e c) Emissão Estimulada de radiação. No processo de absorção o número de fótons do sistema decresce uma unidade após a absorção, enquanto na emissão o número é aumentado por uma unidade. $A$ probabilidade para emissão estimulada é proporcional ao número de fótons presente e o fóton emitido tem coerência de fase com a radiação existente. 
$\hat{O}$ qualquer, dada abaixo [52]:

$$
i \hbar \frac{d \hat{O}}{d t}=[\hat{O}, \hat{H}] .
$$

Como resultado tem-se conjunto de equações não-lineares acopladas dado abaixo:

$$
\begin{aligned}
i \hbar \frac{d \sigma_{z}}{d t} & =2 \gamma \sigma^{+} \hat{a}-2 \gamma^{*} \sigma^{-} \hat{a}^{\dagger} \\
i \hbar \frac{d \sigma^{+}}{d t} & =-\varepsilon_{0} \sigma^{+}+\gamma^{*} \sigma_{z} \hat{a}^{\dagger} \\
i \hbar \frac{d \sigma^{-}}{d t} & =\varepsilon_{0} \sigma^{-}-\gamma \sigma_{z} \hat{a} \\
i \hbar \frac{d \hat{a}}{d t} & =\hbar \omega \hat{a}+\gamma^{*} \sigma^{-} \\
i \hbar \frac{d \hat{a}^{\dagger}}{d t} & =-\hbar \omega \hat{a}^{\dagger}-\gamma \sigma^{+} \\
i \hbar \frac{d \hat{n}}{d t} & =-\gamma \sigma^{+} \hat{a}+\gamma^{*} \sigma^{-} \hat{a}^{\dagger}
\end{aligned}
$$

Curiosamente, a despeito da aparente simplicidade do modelo inicial, soluções exatas de modo geral não são conhecidas. Todavia, podemos apelar para a teoria de perturbações para obter informações a respeito do comportamento desse sistema. Para o problema da interação da luz com a matéria é mais relevante conhecer os valores médios de $d \sigma_{z} / d t$ e $d \hat{n} / d t$. Uma importante conclusão obtida das equações acima é que $d \sigma_{z} / d t=-2 d \hat{n} / d t$, significando que esses operadores não são linearmente independentes e uma dessas equações é redundante. Integrando os valores médios sobre um dado período de tempo $t$ obtemos uma relação entre a variação do valor médio do sistema de dois níveis $\left(\Delta \sigma_{z}=\sigma_{z}(t)-\sigma_{z}(0)\right)$ e a variação do número de fótons $(\Delta n=n(t)-n(0))$, dada por $\left\langle\Delta \sigma_{z} / 2\right\rangle=-\langle\Delta \hat{n}\rangle$. Isto significa que o decréscimo do número de fótons vem acompanhada necessariamente da transição do sistema de dois níveis indo da menor para a maior energia. Essa transição entre os níveis de energia corresponde ao fato de que em um semicondutor tradicional, para cada fóton absorvido deve haver a criação de um par elétron-buraco.

Um modelo totalmente quantizado como o apresentado acima é muito relevante em dispositivos operando com poucos fótons, como detectores de um único fóton ou de números menores que uma centena de fótons, ou em processos transitórios em lasers e fotodetectores. Em dispositivos de conversão fotovoltaica, como as células solares, geralmente o número de fótons é grande o suficiente para permitir uma abordagem clássica do campo eletromagnético. O leitor deve lembrar que em uma célula solar o dispositivo está exposto à densidade de potência produzida pela radiação solar. Portanto, para simplificar o sistema de equações de movimento (13)-(18), devemos substituir os operadores bosônicos $\hat{a}$ e $\hat{a}^{\dagger}$ pelos seus valores médios em um problema com grande número de fótons, transformando-os em variáveis clássicas. Desse modo, é uma boa aproximação fazer $\hat{a} \rightarrow A(t)=A_{0} e^{-i \omega t}$ e $a^{\dagger} \rightarrow A^{*}(t)=A_{0}^{*} e^{i \omega t}$, o que nos leva diretamente a um problema análogo ao do spin $1 / 2$ na presença de um campo magnético variante no tempo e cuja solução apresenta as tão bem conhecidas oscilações de Rabi. A constante $A_{0}$ tem módulo proporcional a $\sqrt{n}$ nessa aproximação, e a densidade de potência da radiação eletromagnética será proporcional a $\left|A_{0}\right|^{2}$, que por sua vez será proporcional ao número de fótons médio $n$ dos sistema, conforme esperado para um problema semi-clássico.

\section{O Coeficiente de Absorção de Luz do Meio Material}

Para o estudo de efeitos fotovoltaicos em células solares estamos interessados diretamente na taxa de absorção de fótons, pois é de se esperar que a cada fóton absorvido um par elétron-buraco seja gerado em um processo maximamente eficiente. A taxa de absorção $W_{i \rightarrow f}$ produzida pela transição entre um estado inicial de menor energia e um estado final de maior energia será calculada utilizando o termo de interação $\hat{H}_{\text {int }}=\gamma \sigma^{+} \hat{a}+\gamma^{*} \sigma^{-} \hat{a}^{\dagger}$ e a regra de ouro de Fermi, dada abaixo [52]:

$$
W_{i \rightarrow f}=\frac{2 \pi}{\hbar}\left|\left\langle f\left|\hat{H}_{\mathrm{int}}\right| i\right\rangle\right|^{2} \delta\left(\sum_{i} E_{i}-\sum_{f} E_{f}\right),
$$

onde $|i\rangle$ denota o estado inicial do sistema e $|f\rangle$ o estado final, $\sum_{i} E_{i}$ é a soma das energias de todos os constituintes do estado inicial e $\sum_{f} E_{f}$ de todos os constituintes do estado final. A função delta de Dirac $\delta\left(\sum_{i} E_{i}-\sum_{f} E_{f}\right)$ garante a conservação de energia no processo. Para que ocorra absorção o estado inicial é dado por $|i\rangle=|\downarrow, n\rangle$ enquanto o estado final apresentará um fóton a menos , ou seja, $|f\rangle=|\uparrow, n-1\rangle$. Aplicando as considerações acima, obtemos o seguinte resultado:

$$
W_{i \rightarrow f}=\frac{2 \pi}{\hbar}|\gamma|^{2} n \delta\left(\varepsilon_{0}-\hbar \omega\right),
$$

uma vez que as somas das energias iniciais e finais são dadas por $\sum_{i} E_{i}=\hbar \omega n-\varepsilon_{0} / 2$ e $\sum_{f} E_{f}=\hbar \omega(n-1)+\varepsilon_{0} / 2$, respectivamente. Tendo em vista a presença da delta de Dirac, nesse sistema idealizado de dois níveis, somente há transição se a energia do fóton for exatamente igual à diferença de energia entre os dois níveis, o que corresponde à presença de uma linha de absorção espectralmente limpa (largura da linha nula). Na prática os efeitos de relaxação, produzidas por vibrações e interações com fônons, por exemplo, produzem uma incerteza $\Delta \omega$ na energia do fóton a ser absorvido e com isso a largura de linha de absorção será não nula.

Agora podemos associar essa taxa de transição ao coeficiente de absorção da radiação eletromagnética, também conhecida como constante de atenuação. A densidade de potência média transportada por uma onda eletromagnética em um meio material é medida através do vetor de Poynting [53]:

$$
\mathbf{S}=\frac{1}{2} \operatorname{Re}\left(\mathbf{E} \times \mathbf{H}^{*}\right),
$$

onde $\mathbf{E}$ e $\mathbf{H}$ são os campos elétrico e magnético da onda, respectivamente, * denota conjugação complexa. Sabe- 
mos que, para ondas planas uniformes, o vetor de Poynting tem relação direta com a densidade de energia eletromagnética $u=\frac{1}{2}\left(\epsilon \mathbf{E}^{2}+\mu \mathbf{H}^{2}\right)$, através da expressão $\mathbf{S} / c=u \hat{\mathbf{n}}$, sendo $c$ a velocidade da luz e $\hat{\mathbf{n}}$ a direção de propagação da radiação. Quando quantizamos a radiação, podemos mostrar que a densidade de energia está associada ao número de fótons, cuja energia individual vale $\hbar \omega$, através da expressão:

$$
u=\frac{S}{c}=\frac{n \hbar \omega}{\Delta V}
$$

de modo que o módulo do vetor de Poynting seja dado por $S=c \hbar \omega n / \Delta V$, onde $\Delta V$ é o volume que contém $n$ fótons. Em meios materiais com perdas, ondas eletromagnéticas planas uniformes propagando-se na direção positiva do eixo $x$ seguem a lei de Lambert de atenuação exponencial para a densidade de potência transportada, na forma:

$$
\frac{d S}{d x}=-\alpha S
$$

permitindo determinar a constante de atenuação $\alpha$ através da expressão:

$$
\alpha=\frac{-\Delta S / \Delta x}{S}\left[\mathrm{~m}^{-1}\right]
$$

Uma vez que um pacote de fótons desloca-se uma distância $\Delta x=c \Delta t$ em um intervalo $\Delta t$ e que $\Delta S$ é proporcional à variação do número de fótons $\Delta n$, que são absorvidos no caminho, temos $\Delta S / \Delta x=\hbar \omega \Delta V^{-1}(\Delta n / \Delta t)$. A taxa de variação no número de fótons $\Delta n / \Delta t$ na presença de um sistema de dois níveis está determinada na equação (20), de modo que podemos escrever a seguinte expressão geral:

$$
\alpha=\frac{W_{i \rightarrow f}}{c}=\frac{2 \pi}{\hbar c}|\gamma|^{2} \delta\left(\varepsilon_{0}-\hbar \omega\right) .
$$

A transição induzida pelos fótons no sistema de dois níveis leva o elétron do estado de menor energia para o de maior energia, deixando o estado fundamental vazio e o estado excitado preenchido. A generalização óbvia é considerar que tenhamos, ao invés de apenas dois níveis discretos de energia, duas bandas de energia, separadas por um gap. Através de absorção de um fóton, o elétron da banda de menor energia, denominada BV, é promovido para a banda de maior energia, previamente desocupada e denominada $\mathrm{BC}$, e pode se deslocar entre níveis permitidos dentro da BC. O nível de energia desocupado na BV também pode ser preenchido por outros elétrons da própria BV. Essa vacância na BV comporta-se como uma partícula de carga positiva denominada buraco (ou lacuna). Portanto, a absorção de fótons é capaz de criar pares elétron-buraco. Uma vez vencida a energia eletrostática de atração entre um par elétron-buraco, que é usualmente pequena em um material semicondutor, essas partículas passam a se movimentar de modo independente e quase livre, aumentando assim a condutividade. As partículas criadas podem também ser espalhadas e eventualmente recombinadas por processos de emissão de luz, denominados processos radiativos ou então através da emissão de fônons, vibrações da rede cristalina do material ou vibrações moleculares, denominados processos não-radiativos.

\subsection{Modelo de bandas materiais semicondutores inorgânicos típicos}

Para modelar as bandas de valência e de condução em semicondutores inorgânicos típicos $[54,55]$, próxima dos pontos de interesse prático em aplicações fotovoltaicas, ou seja, em torno do máximo da BV e do mínimo da BC, devemos lembrar que qualquer função bem comportada $f(x)$ pode ser expandida adequadamente em séries de Taylor, em torno de um ponto $x_{0}$ na forma

$$
f(x)=f\left(x_{0}\right)+\left.\frac{d f}{d x}\right|_{x_{0}}\left(x-x_{0}\right)+\left.\frac{1}{2} \frac{d^{2} f}{d x^{2}}\right|_{x_{0}}\left(x-x_{0}\right)^{2}+\ldots
$$

Se $x_{0}$ é um máximo ou um mínimo da função $f$ então $d f / d x=0$ nesse ponto e $f$ toma a forma parabólica, $f(x)=a+b\left(x-x_{0}\right)^{2}$. Os materiais semicondutores inorgânicos tipicamente tem bandas de energia que podem ser modeladas próximas do mínimo da $\mathrm{BC}$ e do máximo da $\mathrm{BC}$ por funções parabólicas relacionando energia e momento linear, conforme as expressões abaixo:

$$
\begin{aligned}
& E_{B C}(\mathbf{k})=E_{c}+\frac{\hbar^{2}\left(\mathbf{k}-\mathbf{k}_{0}\right)^{2}}{2 m_{c}} \\
& E_{B V}(\mathbf{k})=E_{v}-\frac{\hbar^{2} \mathbf{k}^{2}}{2 m_{v}}
\end{aligned}
$$

onde $E_{c}$ é valor de mínima energia na banda de condução, $E_{v}$ o valor de máxima energia na banda de valência, $\mathbf{k}$ é o vetor de onda associado ao momento $\mathbf{p}=\hbar \mathbf{k}$ da partícula, que parametriza as bandas de energia, $\mathbf{k}_{0}$ é o deslocamento relativo do mínimo da BC. As massas efetivas de elétrons e buracos [54,55], $m_{c}$ e $m_{v}$, respectivamente, são definidas através das expressões $m_{c}=\hbar^{2} /\left[\partial^{2} E /\left.\partial k^{2}\right|_{k=k_{0}}\right]$ e $m_{v}=-\hbar^{2} /\left[\partial^{2} E /\left.\partial k^{2}\right|_{k=0}\right]$.

A Figura 2 ilustra dois tipos de semicondutores, denominados de gap direto $\left(\mathbf{k}_{0}=0\right)$ e gap indireto $\left(\mathbf{k}_{0} \neq 0\right)$. A diferença de energia entre o máximo da banda de valência e o mínimo da banda de condução é denominado bandgap do material, $E_{g}=E_{c}-E_{v}$. Na situação ilustrada na Figura 2.(a) temos um material de gap direto porque a mínima diferença de energia ocorre para um mesmo valor de momento p, enquanto que na Figura 2.(b) o material é dito de gap indireto porque o mínimo da banda de condução não está situado no mesmo valor de momento $\mathbf{p}$ em que encontra-se o máximo da banda de valência.

Para entender o que significa um semicondutor ter gap direto ou indireto é preciso considerar as leis de conservação de energia $\left(\sum_{i} E_{i}=\sum_{f} E_{f}\right)$ e momento linear $\left(\sum_{i} \mathbf{p}_{i}=\sum_{f} \mathbf{p}_{f}\right)$ no processo de absorção do fóton, onde os sub-índice $i$ e $f$ denotam os estados iniciais e finais no processo físico considerado, respectivamente. Devemos lembrar que um fóton transporta energia $E=\hbar \omega$ e momento linear cujo módulo vale $p=\hbar k=E / c$. Uma vez que a velocidade da luz $c$ é muito grande e os fótons 


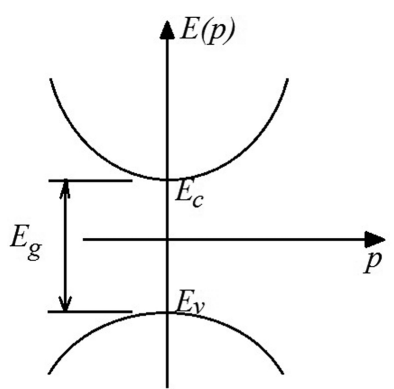

(a)

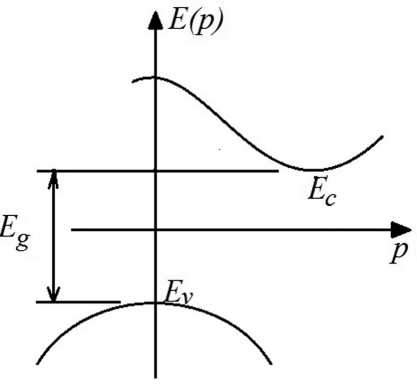

(b)
Figura 2: Estrutura de bandas próxima do mínimo da banda de condução e máximo da banda de valência para semicondutores de gap diret (a) e indireto (b).

não possuem massa, o momento transportado pelo fóton é pequeno. Por exemplo, um fóton de $1 \mathrm{eV}$ transporta $5.33 \times 10^{-28} \mathrm{~kg} . \mathrm{m} / \mathrm{s}$ de momento linear. Por outro lado, um elétron em um cristal tem um número de onda cuja magnitude é proporcional às dimensões da chamada primeira zona de Brillouin, no espaço recíproco, podendo ir de zero até valores nos limites dessa primeira zona de Brillouin. Uma vez que a separação inter-atômica em

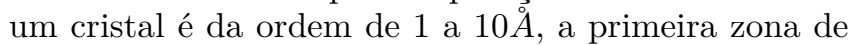
Brillouin terá dimensões máximas na escala de $2 \pi / a$ e um elétron mais próximo das fronteiras da primeira zona de Brillouin terá número de onda $k \sim \pi / a$ e módulo do momento na ordem de $p=\hbar k \sim 10^{-24} \mathrm{~kg} . \mathrm{m} / \mathrm{s}$, que é tipicamente 3 ou 4 ordens de grandeza maior que o momento transportado por um fóton na região do espectro infravermelho ou visível. Nesse caso, num processo envolvendo um par elétron-buraco e um fóton, este último somente contribui para a conservação de energia, mas tem papel irrelevante na conservação de momento, uma vez que a variação de momento para os elétrons e/ou buracos pode ser da ordem de grandeza de $\pi / a$. Um processo de transição direta envolve a absorção de um fóton com a geração de um par elétron-buraco, de tal maneira que o momento linear do sistema seja nulo, ou seja, das leis de conservação de energia e momento temos [4]:

$$
\begin{array}{r}
E_{c}-E_{v}+\frac{\mathbf{p}^{2}}{2 m_{c}}+\frac{\mathbf{p}^{\prime 2}}{2 m_{v}}=E_{g}+\frac{p^{2}}{2 m_{r}}=\hbar \omega, \\
\mathbf{p}+\mathbf{p}^{\prime} \approx 0,
\end{array}
$$

onde $\mathbf{p}$ é o momento de um elétron, $\mathbf{p}^{\prime}$ o momento de um buraco, e $m_{r}$ é a massa reduzida do sistema elétronburaco, definida abaixo:

$$
m_{r}=\frac{m_{c} m_{v}}{m_{c}+m_{v}}
$$

Observe que $|\mathbf{p}|=\left|\mathbf{p}^{\prime}\right|=p$. Desse modo, o fóton de energia $\hbar \omega$ deve fornecer a energia suficiente para vencer o gap $E_{g}$ somada à a energia cinética do par elétron-buraco gerado, sem acrescentar um momento linear apreciável ao sistema.

Um semicondutor de gap direto é aquele para o qual as transições eletrônicas podem ser obtidas a partir da absorção de fótons com energia próxima do valor do gap, $\hbar \omega \sim E_{g}$, sem a assistência de outros processos que permitam conservação de momento. Para um semicondutor de gap indireto, um fóton de energia $\hbar \omega \sim E_{g}$ não é capaz de gerar um par elétron-buraco sem assistência de outros processos porque não possui momento linear suficiente para permitir a conservação de momento linear. O processo assistido envolve a emissão ou absorção de fônons para permitir a conservação de momento linear. As leis de conservação para um semicondutor de gap indireto com absorção de fônon são as seguintes:

$$
\begin{array}{r}
E_{g}+\frac{\left(\mathbf{p}-\mathbf{p}_{0}\right)^{2}}{2 m_{c}}+\frac{\mathbf{p}^{\prime 2}}{2 m_{v}}=\hbar \omega+\hbar \omega_{\mathbf{q}}, \\
\mathbf{p}+\mathbf{p}^{\prime}-\hbar \mathbf{q}=0,
\end{array}
$$

onde $\hbar \omega_{\mathbf{q}}$ é a energia de um fônon com momento $\hbar \mathbf{q}$. O processo envolvendo a emissão de fônon deve satisfazer as seguintes condições:

$$
\begin{array}{r}
E_{g}+\frac{\left(\mathbf{p}-\mathbf{p}_{0}\right)^{2}}{2 m_{c}}+\frac{\mathbf{p}^{\prime 2}}{2 m_{v}}=\hbar \omega-\hbar \omega_{\mathbf{q}}, \\
\mathbf{p}+\mathbf{p}^{\prime}+\hbar \mathbf{q}=0 .
\end{array}
$$

São exemplos de semicondutores de gap direto o arseneto de gálio (GaAs) e o telureto de cádmio ( $\mathrm{CdTe}$ ), enquanto o silício $(\mathrm{Si})$ e o germânio $(\mathrm{Ge})$ tem gap indireto. Tendo em vista que os semicondutores de gap indireto dependem de um processo auxiliar para a absorção fotônica, o coeficiente de absorção é tipicamente reduzido, em relação ao caso dos semicondutores de gap direto. Além disso, uma vez que o número de fônons é altamente dependente da temperatura, através da estatística de Bose-Einstein, os semicondutores de gap indireto tem maior dependência do coeficiente de absorção em relação à temperatura, sendo desse modo menos utilizados na fabricação de dispositivos fotovoltaicos de alta eficiência.

Vamos analisar mais detalhadamente o caso dos semicondutores de gap direto, muito úteis na fabricação de dispositivos optoeletrônicos. Para tanto podemos generalizar a expressão 25 do coeficiente de absorção de um sistema de dois níveis, para o caso de duas bandas de energia em um semicondutor. Nesse caso a diferença de energia do estado fundamental e do estado excitado no sistema de dois níveis, $\varepsilon_{0}$, deve ser substituída por $E_{g}+\frac{\hbar^{2} k^{2}}{2 m_{r}}$, de forma que a função delta de Dirac aparecendo em 25 simplesmente force ao cumprimento da condição de conservação de energia dada por (28). A absorção total corresponderá à soma sobre todos os valores de $\mathbf{k}$, na forma abaixo:

$$
\alpha(\omega)=\sum_{\mathbf{k}} \frac{2 \pi}{\hbar c}|\gamma|^{2} \delta\left(E_{g}+\frac{\hbar^{2} k^{2}}{2 m_{r}}-\hbar \omega\right) .
$$

Admite-se que $\gamma$ seja uma constante em relação à variável $\mathbf{k}$ e nesse caso, transformando o somatório em uma integral através do procedimento $\sum_{\mathbf{k}} \rightarrow=\frac{V o l}{(2 \pi)^{3}} \int d^{3} \mathbf{k}$ obtemos:

$$
\alpha(\omega)=\frac{V o l}{\hbar c \pi}|\gamma|^{2} \int_{0}^{\infty} k^{2} d k \delta\left(E_{g}+\frac{\hbar^{2} k^{2}}{2 m_{r}}-\hbar \omega\right) .
$$


Para podermos integrar a função delta de Dirac devemos realizar a mudança de variáveis $k^{2}=2 m_{r} E / \hbar^{2}$, tal que $2 k d k=2 m_{r} d E / \hbar^{2}$. Um bom exercício é demonstrar o resultado final, dado abaixo:

$$
\alpha(\omega)=\frac{V o l}{2 \pi \hbar c}|\gamma|^{2}\left(\frac{2 m_{r}}{\hbar^{2}}\right)^{3 / 2} \sqrt{\hbar \omega-E_{g}} \Theta\left(\hbar \omega-E_{g}\right) .
$$

Esse comportamento de $\alpha(\omega)$ variando na forma $\sqrt{\hbar \omega-E_{g}}$ é característico de semicondutores de gap direto em condições de bulk [4].

O problema dos semicondutores de gap indireto é um pouco mais intrincado e não será tratado aqui, porque como já mencionado, envolve processos de segunda ordem, com a emissão ou absorção de fótons e fônons, uma vez que transições diretas, envolvendo apenas fótons, são proibidas próximo do gap pela lei de conservação de momento. Entretanto, podemos encontrar o coeficiente de absorção óptica através do uso da regra de ouro de Fermi para transições de segunda ordem. Como é sabido, um processo de segunda ordem tem probabilidade menor de ocorrer do que processos de primeira ordem e por isso semicondutores de gap indireto, como o $\mathrm{Si}$ e o Ge, tem coeficiente de absorção e luminescência pelo menos uma ordem de grandeza menores se comparados a semicondutores de gap direto. Para dispositivos fotovoltaicos que requerem alta eficiência os semicondutores utilizados são de gap direto, e tipicamente envolvem elementos de alta toxicidade ao ser humano, como Te, Cd e As. Em aplicações de larga escala tem sido utilizado o silício policristalino, a despeito do problema do gap indireto, por conta da sua pouca toxicidade e grande abundância.

\section{Estados Localizados e Geração de Éxcitons}

Para completar a análise de absorção em um semicondutor ainda é necessário levar em conta um fenômeno de absorção que ocorre para fótons com energia logo

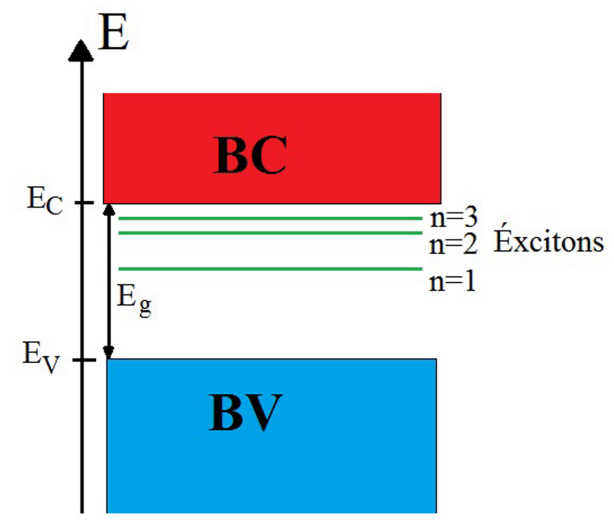

(a) Níveis Excitônicos no Meio do Gap abaixo de $E_{g}$. É sabido da teoria de bandas que não há nenhum estado de energia disponível no meio do gap que seja estendido ao longo do cristal e portanto definido por vetor de onda $\mathbf{k}$. No entanto, é possível, através da absorção de fótons, criar estados elétron-buraco ligados e bem localizados espacialmente no cristal, com energia dentro do gap, mas não definidos por um vetor de onda $\mathbf{k}$. Esses estados de elétron-buraco ligados são denominados éxcitons [56-58]. A Figura 3 ilustra os níveis excitônicos dentro do gap, bem como a representação de um estado de éxciton na rede cristalina, onde um elétron orbita um buraco, formando uma espécie de átomo hidrogenóide.

Os níveis excitônicos de um cristal são obtidos mediante a solução da equação de Schrödinger para um átomo hidrogenóide formado pelo estado elétron-buraco ligado, em que a atração de um elétron por um buraco é descrita pelo potencial coulombiano:

$$
U(r)=-\frac{e^{2}}{4 \pi \varepsilon r},
$$

onde $\varepsilon$ é a permissividade dielétrica do meio material. Em semicondutores típicos $\varepsilon \sim 10 \varepsilon_{0}$. Ao contário do átomo de hidrogênio, onde o núcleo é muito mais massivo do que o elétron, o elétron e o buraco lacuna tem aproximadamente a mesma massa, devendo ser caracterizados pela massa reduzida $m_{r}$, já definida acima. Além disso o éxciton é altamente instável, podendo haver recombinação do par em dado momento, através de emissão de fótons ou outros processos não-radiativos. Os níveis de energia, relativos ao mínimo da banda de condução ficam quantizados e são dados pela expressão abaixo [56-58]:

$$
E_{n}=E_{c}-\frac{m_{r} e^{4}}{2 \hbar^{2}(4 \pi \varepsilon)^{2} n^{2}},
$$

onde $n=1,2,3 \ldots$ é um número inteiro que indexa o número quântico principal do éxciton (não confundir com número de fótons aqui). A criação de um éxciton no estado de energia $E_{n}$ através da absorção de um fóton

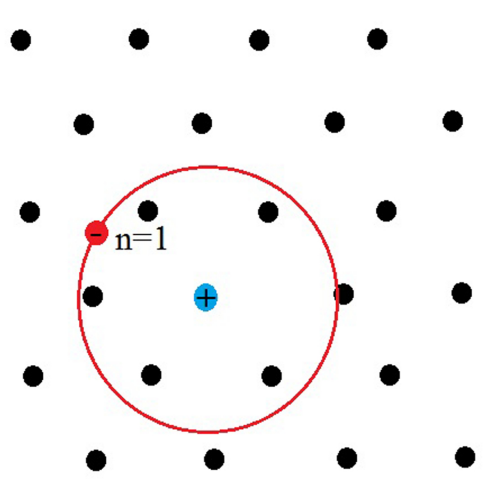

(b) Representação do Éxciton na Rede Cristalina

Figura 3: Níveis excitônicos dentro do gap (a) e a representação de um estado de éxciton na rede cristalina, onde um elétron orbita um buraco, formando uma espécie de átomo hidrogenóide (b). 


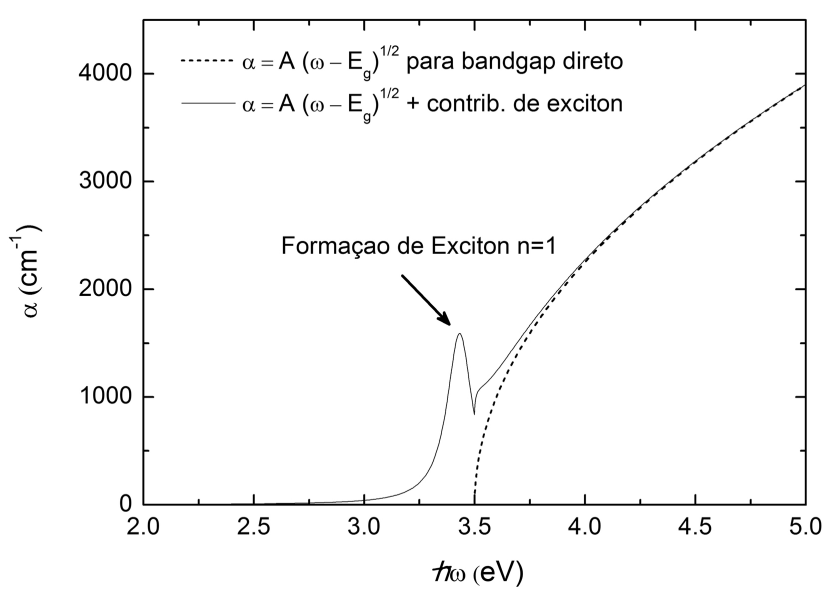

Figura 4: Coeficiente de absorção $\alpha(\omega)$ típico para um material hipotético de gap direto. Os valores adotados foram $E_{g}=3,5 \mathrm{eV}$, $E_{1}=68 \mathrm{meV}, \Gamma=70 \mathrm{meV}$ e $A=3 \times 10^{3} \mathrm{eV}^{-1 / 2} \cdot \mathrm{cm}^{-1}$.

de energia $\hbar \omega$ requer que $\hbar \omega=E_{g}-\frac{m_{r} e^{4}}{2 \hbar^{2}(4 \pi \varepsilon)^{2} n^{2}}$. Como se vê, para criar um éxciton, a energia do fóton pode ser ligeiramente menor do que o gap do material. O exciton que requer a menor energia do fóton corresponde ao nível $n=1$. Para fins de obtermos ordens de grandeza, considere um semicondutor inorgânico típico, em que $\varepsilon \sim$ $10 \varepsilon_{0}$ e $m_{r}=m_{e} / 2$, o que nos da $E_{1}-E_{c}=-68 \mathrm{meV}$ para o éxciton $n=1$, significando que para um semicondutor com $E_{g}=1 \mathrm{eV}$ um fóton com energia $E_{g}-0.068 \mathrm{eV}=$ 0,932eV será absorvido com a criação de um éxciton. Ao coeficiente de absorção $\alpha(\omega)$ dado por (36), para um semicondutor de gap direto, devemos acrescentar a contribuição dos éxcitons. Uma vez que o éxciton é um nível discreto, deveríamos somar um termo da forma (25), $\operatorname{com} \varepsilon_{0}=E_{g}-E_{1}-\hbar \omega$. Na prática efeitos de relaxação suavizam a função delta de Dirac, que pode ser expressa através de uma função lorentziana, lembrando que

$$
\delta(E)=\lim _{\Gamma \rightarrow 0} \frac{1}{\pi} \frac{\Gamma}{E^{2}+\Gamma^{2}},
$$

onde $\Gamma$ é a largura da linha de absorção, fornecendo a seguinte equação:

$$
\begin{aligned}
& \alpha(\omega)=\frac{1}{\pi \hbar c}|\gamma|^{2} \frac{\Gamma}{\left(E_{g}-E_{1}-\hbar \omega\right)^{2}+\Gamma^{2}} \\
& +\frac{V o l}{2 \pi \hbar c}|\gamma|^{2}\left(\frac{2 m_{r}}{\hbar^{2}}\right)^{3 / 2} \sqrt{\hbar \omega-E_{g}} \Theta\left(\hbar \omega-E_{g}\right)
\end{aligned}
$$

A Figura 4 ilustra o coeficiente de absorção para um material de gap direto, levando em conta o nível excitônico $n=1$, utilizando $E_{g}=3,5 \mathrm{eV}, E_{1}=68 \mathrm{meV}, \Gamma=70 \mathrm{meV}$ e $A=\frac{V o l}{2 \pi \hbar c}|\gamma|^{2}\left(\frac{2 m_{r}}{\hbar^{2}}\right)^{3 / 2}=3 \times 10^{3} \mathrm{eV}^{-1 / 2} \cdot \mathrm{cm}^{-1}$.

Os efeitos de excitons de ordem maior e processos envolvendo fônons também devem ser incluidos no cenário, mas geralmente a expressão 39 está em boa concordância com medidas de absorção em semicondutores de gap direto. A geração de portadores está intimamente ligada à taxa de absorção de fótons e ao coeficiente de absorção, evidentemente. Quanto maior o valor do coeficiente de absorção do material maior será o número de portadores fotogerados.

\section{O fenômeno da fotocondutividade}

Um material fotocondutor (ou fotorresistivo) é aquele onde suas propriedades de condutividade elétrica são alteradas na presença de radiação eletromagnética. Tipicamente, significativas variações da condutividade elétrica de um material ocorrem quando a energia dos fótons incidentes é capaz de promover transições de portadores de carga entre bandas de valência e condução. Considere um material semicondutor onde podem concorrer para o transporte de carga os elétrons e os buracos. A densidade de corrente elétrica total $\mathbf{J}$ no material será dada pelas seguintes expressões:

$$
\begin{aligned}
\mathbf{J} & =\mathbf{J}_{e}+\mathbf{J}_{p}, \\
\mathbf{J}_{e} & =e n \mu_{e} \mathbf{E}+e D_{n} \nabla n, \\
\mathbf{J}_{p} & =e p \mu_{p} \mathbf{E}-e D_{p} \nabla p,
\end{aligned}
$$

sendo $e=1,6 \times 10^{-19} \mathrm{C}$ o módulo da carga eletrônica, $p$ é a densidade de buracos, $n$ aqui é a densidade de elétrons, $\mu_{e}$ e $\mu_{p}$ são as mobilidades dos elétrons e buracos, respectivamente, e $D_{n}$ e $D_{p}$ os coeficientes de difusão.

Observe que a densidade de corrente $\mathbf{J}$ pode ser subdividida em dois termos, um para a corrente de elétrons $\mathbf{J}_{e}$ e outro para a corrente de buracos $\mathbf{J}_{\mathbf{p}}$. Por sua vez essas componentes podem ser divididas em um termo de deriva, produzido pelo campo elétrico $\mathbf{E}$ e outro de difusão, produzido pelo gradiente da densidade de portadores. Vamos considerar por simplicidade aqui um material homogêneo, tal que os termos difusivos (de gradiente) possam ser desconsiderados. Considerando a aplicação de um campo externo e a validade da lei de Ohm vetorial, $\mathbf{J}=\sigma \mathbf{E}$, onde $\sigma$ é a condutividade elétrica do material, temos:

$$
\sigma=e n \mu_{e}+e p \mu_{p}
$$

Na ausência de luz (diz-se usualmente no escuro) o material possui $n_{0}$ e $p_{0}$ elétrons e buracos por unidade de volume, respectivamente. $\mathrm{Na}$ presença de luz, o efeito fotoelétrico interno faz o número de portadores variar para $n=n_{0}+\delta n$ e $p=p_{0}+\delta p$. Naturalmente que a fotogeração de pares implica $\delta n=\delta p$. A variação da condutividade pela presença da luz pode ser escrita da seguinte forma, portanto:

$$
\delta \sigma=e \mu_{e} \delta n+e \mu_{p} \delta p .
$$

Vamos analisar a equação que descreve a variação do número de buracos e elétrons na presença de fótons de energia $\hbar \omega$, injetados a uma densidade de potência $S$. Uma vez que não há recombinação se não houverem elétrons e buracos simultaneamente, podemos expressar a taxa de recombinações na forma $R=A n p$, onde $A$ é uma constante do material determinada a partir da regra de ouro de Fermi e denominada de taxa de recombinação. Para pequenas variações em $n$ e $p$ 
podemos desprezar variações da ordem $\delta n \delta p$ e escrever $R=A n_{0} p_{0}+A n_{0} \delta p+A p_{0} \delta n$. Lembrando que em equilíbrio, sem presença de luz, há uma reação contrária de geração na forma $G=A n_{0} p_{0}$ que contrabalança o primeiro termo da taxa de recombinações podemos considerar apenas a taxa $\Delta R=A n_{0} \delta p+A p_{0} \delta n$ nas equações. Lembre ainda que a taxa de recombinações é responsável pelo decréscimo de portadores, ou seja, $\Delta R=-d \delta n / d t=-d \delta p / d t$. Há ainda um termo difusivo capaz de fazer variar a densidade de portadores localmente, e o termo de fotogeração. Desprezando os efeitos difusivos, a fotogeração deve ser proporcional à taxa de absorção de fótons, $\alpha$ e à densidade de radiação eletromagnética incidente $S$. Considerando todos esses ingredientes, obtemos as seguintes equações de taxa:

$$
\frac{\partial \delta p}{\partial t}=\frac{\partial \delta n}{\partial t}=-\frac{\delta n}{\tau_{n}}-\frac{\delta p}{\tau_{p}}+\frac{\alpha S}{\hbar \omega}
$$

onde $\tau_{p}=1 /\left(A n_{0}\right)$ e $\tau_{n}=1 /\left(A p_{0}\right)$ são os tempos de recombinação de buracos e elétrons, respectivamente. Em um material intrínseco (não dopado), $\tau_{p}=\tau_{n}$, mas no caso de haver dopagem esses valores são diferentes, e se reduzem bastante para o portador minoritário, pois aumentam as chances de que esse encontre um portador majoritário e se recombine. De fato, na prática os tempos de recombinação podem variar muito, indo desde a escala de alguns ns até valores próximos de $1 \mathrm{~ms}$ para um mesmo tipo de material, dependendo de diversos fatores, como a temperatura, o grau de cristalinidade do material, a pureza e o aparecimento de defeitos que atuam como armadilhas de cargas. Há contribuições de processos radiativos (quanto há emissão de fótons) e não radiativos, quando a recombinação se dá por outros mecanismos, como emissão de fônons (recombinação térmica) e de Auger (colisão com outros portadores), para o tempo total de recombinação. O tempo de recombinação total é obtido por $\tau=\tau_{r} \tau_{n r} /\left(\tau_{r}+\tau_{n r}\right)$, sendo $\tau_{r}$ e $\tau_{n r}$ os tempos de recombinação radiativo e não radiativo, respectivamente [54]. O tempo de recombinação tipicamente diminui com o aumento da temperatura, devido ao aumento da probabilidade das recombinações não radiativas. Quanto menor o número de defeitos no cristal e maior a cristalinidade, maior será o tempo de recombinação.

É importante ressaltar que a própria densidade de radiação vai depender da profundidade no material considerada, pois deve seguir a lei de Lambert. A dependência do número de fótons com a profundidade induzirá a presença de efeitos difusivos no material, mas tudo isso está sendo negligenciado, por uma questão de simplicidade da análise. Agora queremos resolver o problema em regime estacionário, fazendo $\frac{\partial \delta p}{\partial t}=\frac{\partial \delta n}{\partial t}=0$. Observe que a própria conservação de carga implica que $\delta n=\delta p$. Se não há processos difusivos sendo considerados e tampouco injeção de um único tipo de portador (como poderia ocorrer em uma junção p-n polarizada), então localmente a taxa de variação de elétrons e buracos tem que ser a mesma, sob pena de não conservação local da carga elétrica. Tendo isso em conta, obtém-se:

$$
\delta p=\delta n=\frac{\alpha \tau_{r} S}{\hbar \omega}
$$

sendo $\tau_{r}=\tau_{p} \tau_{n} /\left(\tau_{p}+\tau_{n}\right)$ o tempo de recombinação reduzido dos portadores, e a variação de condutividade do material na presença de luz toma a forma abaixo:

$$
\delta \sigma(\omega)=\frac{e \tau_{r}\left(\mu_{e}+\mu_{p}\right) \alpha(\omega)}{\hbar \omega} S
$$

Em regime linear, a variação de condutividade é diretamente proporcional à densidade de radiação incidente no material, dependendo de uma constante de proporcionalidade que contém informação das propriedades do material. Pode-se observar que a resposta do material é fortemente dependente da frequência dos fótons através de $\alpha(\omega) / \omega$. A variação total da condutividade será dada pela seguinte expressão:

$$
\Delta \sigma=\frac{e \tau_{r}\left(\mu_{e}+\mu_{p}\right)}{\hbar} \int \frac{\alpha(\omega)}{\omega} S(\omega) d \omega
$$

Materiais com boa resposta fotocondutiva são candidatos potenciais para aplicações fotovoltaicas e em dispositivos optoeletrônicos. O fenômeno da fotocondutividade é utilizado amplamente em fotodetectores, medidores de nível de luminosidade e fotorresistores empregados no controle da iluminação pública, por exemplo.

Há alguns materiais com resposta fotocondutiva negativa, fato experimental observado e ainda não muito bem compreendido teoricamente [59-61], e também materiais com efeitos combinados de ordem magnética e fotocondutividade [62].

\section{Modelo equivalente de uma célula fotovoltaica típica}

Uma célula solar eficaz deve contar com os seguintes ingredientes essenciais: i) materiais fotoativos, capazes de absorver a radiação eletromagnética incidente produzindo portadores de carga (elétron-buraco) e ii) um campo elétrico interno forte o suficiente capaz de dissociar os portadores gerados pela luz, antes que esses se recombinem. A descrição detalhada de todos os tipos de células solares utilizando diferentes tipos de materiais e configurações é uma tarefa praticamente impossível e este trabalho não pretende ser exaustivo. Vamos concentrar a atenção nas células solares típicas, que utilizam junções do tipo p-n, que na ausência de luz comportam-se como um diodo normal $[63,64]$.

A junção p-n, ilustrada na Figura 5, apresenta os ingredientes supracitados, pois pode ser construída com semicondutores cuja condutividade seja sensível à presença de luz (tipicamente, todo semicondutor satisfaz essa condição em maior ou menor grau), e pelo fato de haver duas regiões dopadas distintamente, surge uma região internamente no material, denominada de camada de depleção, nas proximidades da interface entre os materiais do tipo p e do tipo $\mathrm{n}$, onde passa a existir um campo 


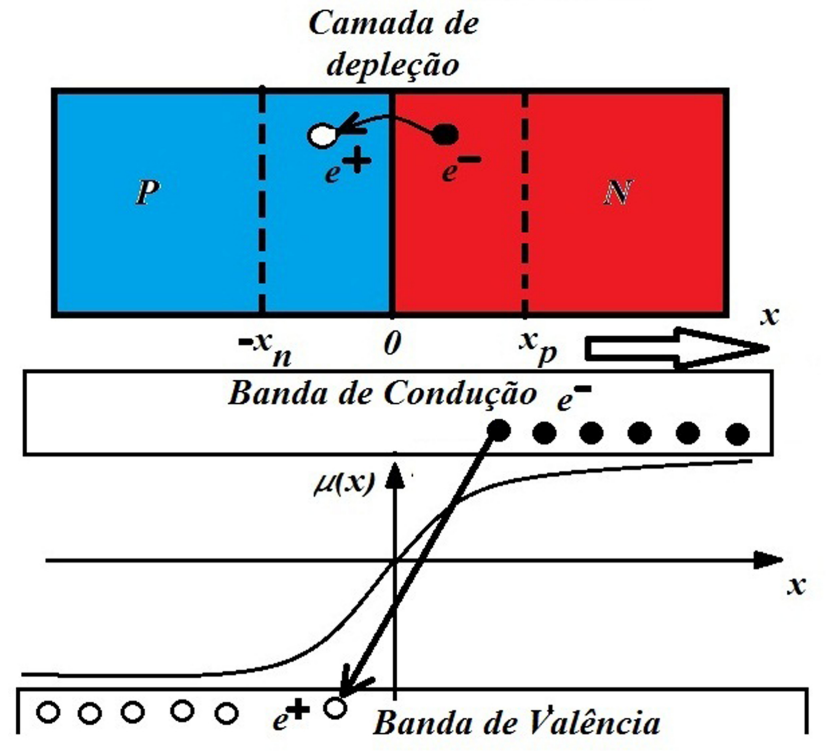

Figura 5: Esquemático de uma junção p-n e perfil do potencial químico $\mu(x)$ ao longo do dispositivo. Próximo da região de contato entre o lado $\mathrm{p}$ e o lado $\mathrm{n}$, os elétrons do lado $\mathrm{n}$ migram para o lado $\mathrm{p}$, deixando o lado $\mathrm{n}$ positivamente carregado $\mathrm{e}$ o lado $\mathrm{p}$ negativamente carregado, o que produz um campo elétrico interno na região da camada de depleção de portadores.

elétrico interno muito intenso, que é capaz de produzir a dissociação dos portadores gerados localmente, levando elétrons para um lado e buracos para o outro lado da junção. Nesse caso os buracos serão conduzidos para o lado p e os elétrons fotogerados para o lado $n$ da junção. A tensão elétrica fotogerada em circuito aberto será dependente essencialmente da diferença entre os potenciais químicos do lado $\mathrm{p}$ e do lado $\mathrm{n}$.

A relação entre tensão e corrente em um diodo convencional é dada pela seguinte equação $[54,55,58]$ :

$$
J(V)=J_{s}\left(e^{V / V_{T}}-1\right),
$$

onde $J(V)$ é a densidade de corrente através da junção, $V$ é a diferença de potencial entre os terminais da junção e $V_{T}=k_{B} T / e$ é o potencial equivalente térmico, sendo $k_{B}$ a constante de Boltzmann e $T$ a temperatura absoluta. Em temperatura ambiente, esse valor é tomado como $26 \mathrm{mV}$. Para $V \rightarrow-\infty$ resta apenas uma densidade de corrente denominada de saturação reversa, $J_{s}$, dada pela seguinte expressão [58]:

$$
J_{s}=\frac{e D_{p} n_{i}^{2}}{N_{D} L_{p}}+\frac{e D_{n} n_{i}^{2}}{N_{A} L_{n}},
$$

onde $D_{n}$ e $D_{p}$ são as constantes de difusão de elétrons e buracos, respectivamente, $n_{i}$ a densidade intrínseca de portadores do material na temperatura $T, N_{D}$ a densidade de impurezas de dopagem doadoras (tipo n) e $N_{A}$ o número de impurezas aceitadoras (tipo p), $L_{p}=\sqrt{D_{p} \tau_{p}}$ e $L_{n}=\sqrt{D_{n} \tau_{n}}$ são os livres caminhos médios de buracos e elétrons antes da recombinação, respectivamente.

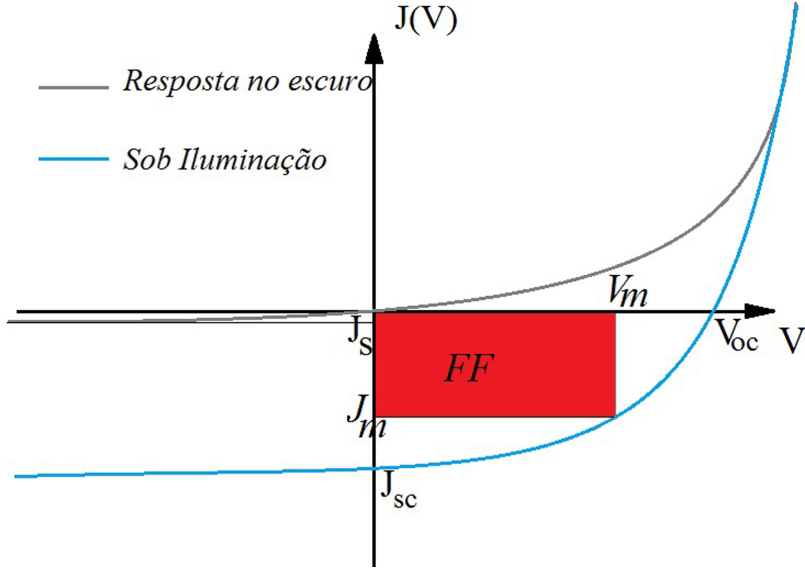

Figura 6: Resposta típica de uma célula fotovoltaica no escuro e sob iluminação. $V_{o c}$ e $J_{s c}$ são a tensão de circuito aberto e a densidade de corrente de curto circuito, respectivamente, $J_{s}$ é a densidade de corrente de saturação reversa, no escuro, $V_{m}$ e $J_{m}$ são a tensão e corrente de máxima transferência de potência possível e o fator de preenchimento $F F$ é calculado por $V_{m} J_{m} /\left(V_{o c} J_{s c}\right)$.

Na presença de luz, há uma corrente fotogerada que deve ser considerada no cenário:

$$
J(V)=J_{s}\left(e^{V / V_{T}}-1\right)-J_{L},
$$

onde $J_{L}$ é proporcional à densidade de potência luminosa $S$ incidente na junção.

Alguns parâmetros elétricos são fundamentais na caracterização de células solares, independentemente do tipo de dispositivo e dos materiais utilizados, a saber: i) tensão de circuito aberto, medida com corrente elétrica nula na presença de luz, ii) corrente de curto circuito, que é obtida medindo-se a corrente fotogerada com os terminais da célula em curto-circuito na presença de luz, iii) eficiência de conversão fotovoltaica e iv) fator de preenchimento. Todos esses devem ser medidos sob iluminação com uma fonte padrão capaz de simular a distribuição espectral presente na radiação solar. Esse padrão tipicamente empregado é obtido utilizado uma fonte de luz AM 1,5 (Air Mass 1,5). O padrão $A M$ leva em conta o caminho que a luz solar necessita percorrer para chegar à superfície da Terra e é dado pela fórmula $A M \approx 1 / \cos z$, onde $z$ é o ângulo de zenite [63]. O padrão 1,5 corresponde à intensidade de radiação solar para a latitude de $48,2^{\circ}$ e é o mais universalmente aceito na caracterização de células solares.

A Figura 6 ajuda a compreender melhor os parâmetros de caracterização de uma célula solar. O fator de preenchimento é dado, em valores percentuais, pela seguinte expressão:

$$
F F(\%)=100 \times \frac{V_{m} J_{m}}{V_{o c} J_{s c}},
$$

onde $V_{o c}$ e $J_{s c}$ são a tensão de circuito aberto e a densidade de corrente de curto circuito, respectivamente, $V_{m}$ e $J_{m}$ são a tensão e corrente de máxima transferência de potência possível para uma carga resistiva conectada aos 


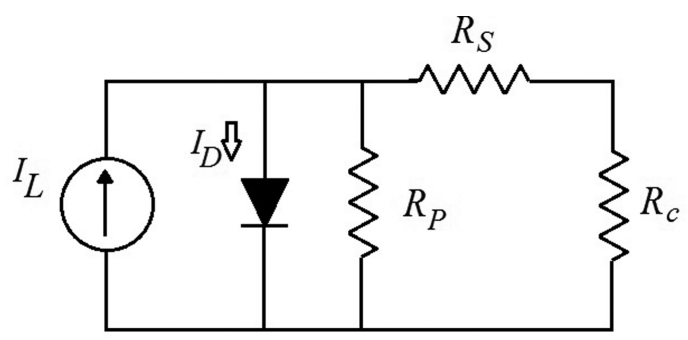

Figura 7: Circuito equivalente de uma célula fotovoltaica típica: $I_{L}=J_{L} A_{e}$ representa a fonte de corrente fotogerada no dispositivo de área $A_{e}$ submetida à luz, $I_{D}$ representa o diodo ideal que seria observado no escuro, $R_{S}$ é o resistor em série e $R_{P}$ o resistor em paralelo, representando desvios da idealidade do dispositivo ideal, enquanto $R_{c}$ representa uma resistência de carga para a qual potência deve ser transferida.

terminais da célula. A eficiência de conversão fotovoltaica externa $\eta_{e}$ é dada por

$$
\eta_{e}(\%)=100 \times \frac{V_{m} J_{m}}{S}=F F \frac{V_{o c} J_{s c}}{S},
$$

onde $S$ é a densidade de potência da radiação incidente no dispositivo.

Da equação (51), podemos determinar a tensão de circuito aberto fazendo $J\left(V_{o c}\right)=0$, o que produz o seguinte resultado:

$$
V_{o c}=V_{T} \ln \left(\frac{J_{L}}{J_{s}}+1\right)
$$

enquanto que para $V=0$ temos a corrente de curto circuito, $J_{s c}=-J_{s}-J_{L} \approx-J_{L}>>J_{s}$.

Outros parâmetros como a resistência série e paralelo dos dispositivos fotovoltaicos são dependentes do modelo equivalente empregado, mas também são bastante úteis e serão discutidos a seguir. A Figura 7 mostra o circuito equivalente tipicamente utilizado [64].

Nesse modelo a corrente fotogerada é representada por uma fonte de corrente $I_{L}=J_{L} A_{e}$, sendo $A_{e}$ a área de seção transversal do dispositivo, e que é submetida à luz, $I_{D}$ é o termo de diodo ideal no escuro. No modelo o resistor em paralelo $R_{P}$ representa fuga de corrente por efeitos de recombinação interna indesejáveis, em centros de armadilhamento de cargas ou mesmo em curtocircuito interno no dispositivo, provenientes de falhas na fabricação. Idealmente tem-se que $R_{P} \rightarrow \infty$. Já o resistor em série $R_{s}$ representa o efeito Joule ao longo do próprio dispositivo, produzido por exemplo por armadilhas de carga e outras barreiras. A resistência em série tipicamente aumenta com o aumento da espessura das camadas semicondutoras do dispositivos. No caso ideal $R_{s} \rightarrow 0$. Finalmente, $R_{c}$ é um resistor de carga para o qual se deseja transferir a potência fotogerada na célula fotovoltaica.

Uma forma simplificada de determinar os valores de resistência série e paralelo que entram no modelo em um dispositivo fotovoltaico a partir da curva experimental $I \times V$, que é usualmente similar à apresentada na Figura
6, é a seguinte [65]:

$$
\begin{aligned}
& R_{s}=\left.\frac{d V}{d I}\right|_{I=0}, \\
& R_{p}=\left.\frac{d V}{d I}\right|_{V=0},
\end{aligned}
$$

Se $R_{s}$ é grande e $R_{p}$ é um valor relativamente pequeno, ou seja, fogem da idealidade, a curva de resposta sob iluminação tende a uma reta, produzindo um fator de preenchimento baixo, o que corresponde a um mau funcionamento do dispositivo.

\section{Principais tipos de células e materiais utilizados}

Na presente Seção serão descritos suscintamente os principais tipos de células fotovoltaicas e os diversos materiais empregados na sua fabricação. Cada tipo possui aplicações e performance específicas. As células fotovoltaicas tem sido classificadas em três gerações, categorizadas conforme os materiais utilizados, métodos de processamento adotados e nível de maturidade comercial $[12,66]$. Normalmente as células solares recebem seus nomes de acordo com o semicondutor selecionado para a aplicação nos dispositivos [67]. Dentre os diferentes tipos de células encontram-se os dispositivos fabricados com silício, semicondutores orgânicos, telureto de cádmio (CdTe), pontos quânticos, perovskitas e outros. Apesar de algumas desvantagens, como a falta de flexibilidade mecânica, a primeira geração baseada em silício, ainda é dominante no mundo todo há décadas, principalmente pela relativamente alta eficiência de conversão de energia (ECE), geralmente de 15 a $20 \%[68,69]$.

Usualmente, a seleção dos materiais para aplicação em células solares depende de características como coeficiente de absorção, discutido anteriormente na presente contribuição, band gap, toxicidade, estabilidade e disponibilidade. Semicondutores comumente utilizados são aqueles que apresentam band gap entre 1,1 e 1,7 eV, com o intuito de respeitar o limite de Shockley-Queisser [70], que prevê máxima eficiência de $44 \%$ para um valor de $E_{g} /\left(k_{B} T_{s}\right) \approx 2$, onde $E_{g}$ é o valor do gap e $T_{s}$ a temperatura de corpo negro do corpo radiante (aqui é relevante o sol, $\left.T_{s} \approx 6000 \mathrm{~K}\right)$. Todavia, semicondutores com band gaps maiores também são utilizados.

Além disso, é necessário que os materiais adotados tenham bons coeficientes de absorção para fins de obter melhor eficiência na conversão de luz solar em energia elétrica [12]. Do ponto de vista prático é necessário levar em conta ainda as características de cada tecnologia e as condições ambientais às quais os dispositivos serão expostos.

\subsection{Primeira Geração - Wafers de Silício}

A primeira geração consiste em células solares baseadas em wafers de silício e são as mais comumente comercializadas, podendo representar $90 \%$ do mercado dos 
dispositivos fotovoltaicos, além de apresentarem vantagens como boa eficiência e desempenho em longo prazo. Este grupo de células de silício podem ser divididas em duas categorias, monocristalinos e policristalinos. Esta geração é a tecnologia de fotovoltaicos mais antiga e mais popular devido a sua alta eficiência de conversão $[12,66]$.

O silício é um material facilmente encontrado, sendo o segundo mais abundante na crosta terrestre (o primeiro é o oxigênio, pois a crosta é formada majoritariamente por óxidos metálicos), e em geral apresenta boa estabilidade e não toxicidade, além de um band gap em torno de 1,12 eV, o que o torna ideal para utilização em células solares [66].

Células que utilizam o Si na sua forma monocristalina exibem melhor desempenho em relação aos dispositivos utilizando Si policristalino, apresentando eficiências de conversão entre $17 \%$ e $27 \%$. Todavia, os custos de manufatura são mais altos, devido à alta pureza requerida no processamento dos materiais [66].

Dispositivos fotovoltaicos produzidos com silício policristalino apresentam menores custos de processamento, com consequente redução da eficiência, de $12 \%$ a $20 \%$, quando comparados à células de Si monocristalino. Essa tecnologia não depende de altos níveis de pureza dos materiais ou de cristais perfeitos. Há inclusive uma classificação da pureza do Si de acordo com a aplicação, denominadas de pureza de grau eletrônico (para fins de uso na eletrônica, que requer pureza maior que 99, 9999999\%) e grau solar (pureza de 99,9999\% para fabricação de células e painéis solares) e grau metalúrgico.

\subsection{Segunda Geração - Filmes Finos}

A segunda geração se caracteriza por painéis confeccionados na forma de filmes finos, o que gera maior economia de material. Os dispositivos desta geração são reconhecidos por fornecerem flexibilidade, facilidade de instalação, tempo de vida médio de 25 anos e baixo custo de processamento, mas tipicamente apresentam eficiências mais baixas. Com camadas de absorção de luz em torno de $1 \mu \mathrm{m}$, células de filme fino podem ser obtidas com materiais como Si amorfo, CdTe e composições dos elementos cobre, índio, gálio e selênio (CIGS) $[12,66]$.

\subsubsection{Silício Amorfo}

Na segunda geração, as células de Si amorfo são as primeiras fabricadas em escala industrial, e por isso as mais bem desenvolvidas da segunda geração, estando presentes comercialmente há mais de 15 anos [66]. São processadas em temperaturas relativamente baixas, o que permite o uso de diferentes substratos, incluindo polímeros e outros materiais flexíveis [66]. Os principais problemas são a baixa eficiência de conversão, que para células comerciais se encontra entre $4 \%$ e $8 \%$. Isso se deve à presença maior de defeitos e armadilhas de carga em um material amorfo, o que reduz a mobilidade dos portadores e aumenta a taxa de recombinações indesejáveis em um dispositivo fotovoltaico. Exemplos de aplicações práticas para células de silício amorfo são calculadoras e alimentação de casas, prédios e instalações remotas [67].

\subsubsection{Telureto de Cádmio}

As células de CdTe apresentam considerável desempenho, com eficiências em torno de $9 \%$ e $22,1 \%$ e coeficiente de absorção de $5 \times 10^{1} 5 / \mathrm{cm}$, sem contar facilidade e baixo custo de processamento. No entanto, o cádmio é um metal pesado e, portanto, representa riscos devido a sua toxicidade, assim como aumento de custos para sua reciclagem $[66,71]$, apesar de o composto CdTe exibir características interessantes para aplicação em dispositivos fotovoltaicos, como por exemplo o band gap direto de aproximadamente $1,5 \mathrm{eV}$.

\subsubsection{CIGS}

Os compostos de CIGS são semicondutores com alto coeficiente de absorção, o que quer dizer que camadas mais finas do material podem ser incorporadas na composição das células solares sem afetar a eficiência dos dispositivos. Células que apresentam o composto CIGS costumam exibir eficiências de $10 \%$ a 22,6\% [67,71]. Através de camadas semicondutoras menos espessas é possível realizar a deposição dos materiais em substratos flexíveis. Por outro lado, as técnicas empregadas usualmente dependem de altas temperaturas e podem reduzir as possibilidades de utilização de materiais, como polímeros orgânicos. O CIGS pode ser depositado através de técnicas comuns como sputtering, evaporação, impressão e deposição por feixe de elétrons. Como principais vantagens deste tipo de célula podemos citar o longo tempo de vida útil, baixa degradação e possibilidade de criar dispositivos flexíveis e leves [66].

\subsection{Terceira Geração - Novas Tecnologias}

A terceira geração de painéis solares compreende as novas tecnologias, que estão sendo demonstradas ou desenvolvidas mas não necessariamente disponíveis em escala industriale. Entre a variedade de dispositivos desta geração, encontram-se células com nanocristais, polímeros, corantes e perovskitas $[66,67]$. Por apresentarem materiais alternativos, configurações de dispositivos e técnicas de fabricação ainda em fase de desenvolvimento, as células que pertencem a esta geração comumente exibem eficiências mais baixas quando comparadas às tecnologias anteriores. No entanto, oferecem diversas vantagens, tais como flexibilidade mecânica, baixo custo e facilidade de processamento.

\subsubsection{Células de Nanocristais}

Também chamados de células solares de pontos quânticos, são dispositivos que consistem de semicondutores com escalas de nanocristais. Materiais como silício poroso ou dióxido de titânio $\left(\mathrm{TiO}_{2}\right)$ poroso são frequentemente utilizados em células de pontos quânticos[66]. 


\subsubsection{Células Solares Orgânicas}

Células solares orgânicas são constituídas de filmes finos de semicondutores orgânicos, como pequenas moléculas e polímeros condutores e podem apresentar eficiências de conversão em torno de 10\% [67]. Em uma direção, dispositivos fotovoltaicos tem sido obtidos com pequenas moléculas como a Ftalocianina de Cobre $(\mathrm{CuPc})$ e o Pentaceno[12] fazendo o papel de camada semicondutora, depositada por meio de evaporação térmica em condições que exigem vácuo.

Seguindo outro caminho, os polímeros conjugados atraem enorme interesse como camada semicondutora devido às suas características de absorção, flexibilidade mecânica, baixo peso molecular, compatibilidade com diversos substratos, solubilidade em vários solventes e, baixo custo e facilidade de processamento, já que podem ser depositados através de técnicas simples como spin coating ou mesmo casting e por não dependerem de altas temperaturas de processamento [72]. O Poli(3-hexiltiofeno) (P3HT) e o PEDOT:PSS são exemplos de polímeros condutores utilizados em células solares $[39,40]$.

É importante mencionar que as densidades de estados eletrônicos de materiais orgânicos não apresentam uma estrutura de bandas que podem ser expressas por funções parabólicas, como é o caso dos semicondutores inorgânicos cristalinos, pois as cadeias poliméricas apresentam estados discretos de energia, que dependem do comprimento da cadeia, e que são suavizados pelas vibrações excitadas por temperatura. Na média as densidades de estados dos orbitais moleculares de maior energia ocupados (do inglês Highest Occupied Molecular Orbitals - HOMO) correspondem a uma banda de valência, enquanto as densidades de estados dos orbitais moleculares de menor energia vazios (do inglês Lowest Unoccupied Molecular Orbitals - LUMO) correspondem a uma banda de condução dos polímeros orgânicos, e são melhor descritos por funções gaussianas, da forma $D(E)=D_{0} e^{-\left(E-E_{0}\right)^{2} / \sigma^{2}}$, sendo $D_{0}$ uma constante, $E_{0}$ a energia no centro da banda (do HOMO ou do LUMO) e a constante $\sigma$ associada à largura da banda. Devido a maior números de defeitos e ligações pendentes nas cadeias poliméricas, é comum o aparecimento de estados localizados, de defeitos, entre o HOMO e o LUMO, que atuam como armadilhas de carga e reduzem a mobilidade dos portadores.

\subsubsection{Células Híbridas}

Células solares híbridas caracterizam-se pela heterojunção orgânico/inorgânico, integrando propriedades de semicondutores inorgânicos e orgânicos. Este tipo de dispositivo adota materiais orgânicos, no caso polímeros conjugados que absorvem luz, para atuarem como camada doadora e para transporte de lacunas. Já os materiais inorgânicos são utilizados como camada aceitadora e para transporte de elétrons [67]. Exemplos de materiais que podem ser utilizados neste tipo de dispositivo são o P3HT como camada doadora e $\mathrm{TiO}_{2}$ como camada aceitadora $[39,40]$.
Dispositivos híbridos têm potencial não só para baixo custo por processos roll-to-roll, mas também para conversão de energia solar em alta escala [67].

\subsubsection{Células Sensibilizadas por Corantes (DSSC)}

Dentre as células solares baseadas em material orgânico, as células sensibilizadas por corantes (do inglêns DyeSensitized Solar Cell - DSSC), também conhecidas como células de Grätzel [73], são o único tipo com implantação comercial significativa, como instalações capazes de gerar até $2000 \mathrm{KWh}$ por ano na Suíça [74]. A estrutura convencional das DSSCs, consiste de substrato de vidro condutivo, um eletrodo semicondutor de óxido metálico sensibilizado por corante, um eletrodo contador de catalisador, e uma solução eletrolítica inserida entre os dois eletrodos. Os dispositivos sensibilizados por corantes como o rutênio e as porfirinas apresentam eficiências promissoras, em torno de $7 \%$ a $13 \%$, bem como estabilidade a longo termo. Um dos materiais mais comuns para aplicação neste tipo de fotovoltaico é o dióxido de titânio, e elementos como fluoreno e cumarina passaram a ser utilizados como parte do esforço de criar corantes orgânicos livres de metais pesados [12]. DSSCs podem ser utilizadas para gerar energia tanto em ambientes internos quanto externos, ou seja, este tipo de célula é capaz de converter luz de diversas fontes, sendo elas artificiais ou naturais [67].

\subsubsection{Células de Perovskita}

Este tipo de dispositivo originou-se das DSSCs e utilizam perovskita como agente sensibilizador. A perovskita representa uma classe de compostos $\mathrm{ABX}_{3}$, em que $\mathrm{X}$ é um halogênio $\left(\mathrm{I}^{-}, \mathrm{Br}^{-}\right.$e $\left.\mathrm{Cl}^{-}\right)$e, $\mathrm{A}$ e B são cátions de diferentes tamanhos [75]. Células obtidas com este tipo de material podem apresentar eficiências de conversão de $22,1 \%$, recorde estabelecido em 2018 [71], oferecendo vantagem sobre os fotovoltaicos convencionais. No entanto, ainda há questões com relação a estabilidade e durabilidade dos dispositivos, já que o material degrada com o tempo e, portanto, apresenta queda na eficiência [66]. Além disso, é preciso eleger elementos e processos de baixo custo, bem como evitar materiais raros e tóxicos, a exemplo do chumbo, um dos elementos chave da perovskita, que apesar de ser abundante, exibe riscos com relação a toxicidade [75].

\subsection{Células Tandem ou de Múltiplas Junções}

Reconhecidas como células com várias camadas, podem ser compostas por uma variedade de materiais. A técnica de empilhar múltiplas junções passou a ser utilizada para evitar altas taxas de degradação dos materiais sem afetar a eficiência dos dispositivos, isso porque seria necessário diminuir a espessura das camadas para evitar degradação, mas quanto menor a camada, menor a absorção de luz, e portanto, menor a eficiência de conversão. Sendo assim, o uso de células tandem passou a ser disseminado não 
só para fins de evitar degradação, mas para melhorar as taxas de conversão e aumentar a eficiência dos dispositivos, podendo alcançar marcas de até 40\% [67]. Por outro lado, a melhora no desempenhos dos fotovoltaicos de múltiplas junções compensa nos custos de obtenção das células, já que o processamento é mais complexo e consequentemente mais caro. A relação entre custo e performance limita o número de aplicações práticas destes dispositivos, podendo ser utilizados principalmente no setor aeroespacial [67].

\section{5. Óxidos Metálicos Semicondutores}

Vários materiais óxidos metálicos, como $\mathrm{TiO}_{2}, \mathrm{ZnO}$, $\mathrm{SnO}_{2}, \mathrm{SrTiO}_{3}, \mathrm{Zn}_{2} 2 \mathrm{SnO}_{4}, \mathrm{WO}_{3}$ e $\mathrm{Nb}_{2} \mathrm{O}_{5}$ têm sido utilizados, sobretudo como anodo fotoativo, atuando como um coletor de elétrons. Essa é uma área ativa de estudos, que concentram-se na obtenção de materiais nanoestruturados. Tipicamente, o desempenho geral dos dispositivos fabricados dependem da forma, tamanho, morfologia e band-gap do material nanoestruturado. Os óxidos metálicos como os citados possuem band-gaps superiores a $3 \mathrm{eV}$ e apresentam resistência à corrosão e propriedades eletrônicas consideradas interessantes [76].

Atualmente, o semicondutor $\mathrm{TiO}_{2}$ é considerado o melhor material para o eletrodo foto anódico para CSSC, e a célula a base de $\mathrm{TiO}_{2}$ mostrou ECE de 14,5\% [77]. Além disso, o $\mathrm{TiO}_{2}$ é um óxido metálico de baixo custo, não tóxico e amplamente disponível. Como alternativa ao $\mathrm{TiO} 2$, pode-se citar o óxido de zinco $(\mathrm{ZnO})$, que possui propriedades eletrônicas semelhantes, e mostra um pouco mais de mobilidade de elétrons do que o $\mathrm{TiO}_{2}$. Utilizando o $\mathrm{TiO}_{2}$, um trabalho descrevendo a fabricação de células solares do tipo Grätzel e fazendo a análise do seu funcionamento já foi publicado na Revista Brasileira de Ensino de Física [78], e anteriormente ainda já havia um trabalho relatando a confecção e caracterização de células solares caseiras [79].

\section{Conclusões}

Sumarizando, no presente trabalho, utilizando-se do modelo de sistema de dois níveis acoplado a um campo de fótons, foram apresentados os princípios físicos da conversão fotovoltaica de energia. O problema spin-bóson foi utilizado de forma pedagógica para servir como um exercício contextualizado de mecânica quântica e física dos semicondutores. A obtenção do coeficiente de absorção luminosa nos meios materiais foi demonstrada a partir da regra de ouro de Fermi e o problema dos semicondutores de gap direto e indireto também foi discutido, considerando-se a conservação de energia e momento linear das partículas envolvidas. Os estados localizados e a geração de éxcitons também foram considerados, através de um modelo de átomo hidrogenóide. Em um tratamento clássico, o fenômeno da fotocondutividade foi apresentado simplificadamente e o modelo equivalente de uma célula fotovoltaica convencional, com os principais parâmetros de caracterização experimental de uma célula solar foram apresentados, para finalmente ser apresentado um panorama geral dos tipos de células solares e materiais utilizados na sua construção. Acredita-se que o presente trabalho possa servir de guia para estudantes e pesquisadores interessados na área da fotovoltaica, mas de forma alguma pretende ser exaustivo e muitos tópicos relevantes não puderam ser abordados.

\section{Agradecimentos}

C.A. Dartora agradece ao $\mathrm{CNPq}$, pelo suporte financeiro parcial, através de Bolsas de Produtividade em Pesquisa CNPq 301848/2017-3. O presente trabalho foi realizado com apoio da Coordenação de Aperfeiçoamento de Pessoal de Nível Superior - Brasil (CAPES) - Código de Financiamento 001.

\section{Referências}

[1] E. Becquerel, Comptes Rendus. 9, 561 (1839).

[2] C.E. Fritts, American Journal of Science 26, 465 (1883).

[3] R.S. Ohl, US Patent 2402662A (1941).

[4] J.I. Pankove, Optical Processes in Semiconductors (Dover Books on Physics, Chicago, 2010).

[5] R.M. Swanson, Science 324, 891 (2009).

[6] M. Bazilian, I. Onyeji, M. Liebreich, I. MacGill, J. Chase, J. Shah, D. Gielen, D. Arent, D. Landfear e S. Zhengrong, Renewable Energy 53, 329 (2013).

[7] M.Z. Jacobson, Energy \& Environmental Science 2, 148 (2009).

[8] B. Parida, S. Iniyan e R. Goic, Renewable and Sustainable Energy Reviews 15, 1625 (2011).

[9] S. Rehman, B. Maher e A. Said, Renewable and Sustainable Energy Reviews 11, 1843 (2007).

[10] R.W. Miles, Vacuum 80, 1090 (2006).

[11] L. Hernández-Callejo, S. Gallardo-Saavedra e V. AlonsoGómez, Solar Energy 188, 426 (2019).

[12] P.G.V. Sampaio e M.O.A. González, Renewable and Sustainable Energy Reviews 74, 590 (2017).

[13] M.H. Alaaeddin, S.M. Sapuan, M.Y.M. Zuhri, E.S. Zainudin e F.M. AL-Oqla, Renewable and Sustainable Energy Reviews 102, 318 (2019).

[14] R. von Baltz e W. Kraut, Phys. Rev. B 23, 5590 (1981).

[15] G.A. Buxton e N. Clarke, Phys. Rev. B 74, 085207 (2006).

[16] U. Aeberhard e U. Rau, Phys. Rev. Lett. 118, 247702 (2017).

[17] W. Norwood Lowry, Phys. Rev. 35, 1270 (1930).

[18] E. Tselepis, E. Fortin e A. Mysyrowicz, Phys. Rev. Lett. 59, 2107 (1987).

[19] S.M. Young, F. Zheng e A.M. Rappe, Phys. Rev. Lett. 109, 236601 (2012).

[20] D. Daranciang, M.J. Highland, H. Wen, S.M. Young, N.C. Brandt, H.Y. Hwang, M. Vattilana, M. Nicoul, F. Quirin, J. Goodfellow et al., Phys. Rev. Lett. 108, 087601 (2012).

[21] Q. Hoarau e Y. Perez, Renewable and Sustainable Energy Reviews 94, 510 (2018).

[22] E.T. Hu, G.Q. Yue, R.J. Zhang, Y.X. Zheng e S.Y. Wang, Renewable Energy 77, 442 (2015). 
[23] L. Chiodo, Current Opinion in Green and Sustainable Chemistry 17, 49 (2019).

[24] E. Tatsi e G. Griffini, Solar Energy Materials and Solar Cells 196, 43 (2019).

[25] C.K. Chiang, C.R. Finche, Y.W. Park, A.J. Heeger, H. Shirakawa, E.J. Lois, C.S. Gau, A.G. MacDiarmid, Phys. Rev.Lett. 39, 1098 (1977).

[26] S. Glenis, G. Tourillon e F. Garnier, Thin Solid Films 139, 226 (1986).

[27] G. Horowitz, Advanced Materials 2, 287 (1990).

[28] N.S. Sariciftci, L. Smilowitz, A.J. Heeger e F. Wuld, Science 258, 1474 (1992).

[29] G. Gustafsson, Y. Cao, G.M. Treacy, F.K. Lavetter, N. Colanere e A.J. Heeger, Nature 357 477, (1992).

[30] N. Tessler e Y. Roichman, Organic Electronics 6, 200 (2005).

[31] D. Maia, M.A. De Paoli, O.L. Alves, A.J.G. Zarbin e S. Neves, Química Nova 23, 204 (2000).

[32] L.S. Berlim, F. Thomazi, E. Burkarter, C.E.B. Marino, C.K. Saul, A.S. Ito e C.A. Dartora, Current Nanoscience 14, 1 (2018).

[33] N. Tessler, N.T. Harrison and R.H. Friend, Adv. Materials 10, 64 (1998).

[34] Z. Xu, L.M. Chen, G.W. Yang, C.H. Huang, J.H. Hou, Y. Wu, G. Li, C.S. Hsu e Y. Yang, Adv. Funct. Mater. 19, 1227 (2009).

[35] Z. Chu, F.G. Chang, Z.Y. Liu e L. Han, Journal of Alloys and Compounds 793, 662 (2019).

[36] R. Steim, P. Schilinsky, S.A. Choulis, e C.J. Brabec, Solar Energy Materials and Solar Cells 93, 1963 (2009).

[37] G. Yu e A.J. Heeger, J. Appl. Phys. 78, 4510 (1995).

[38] L.S. Roman, W. Mammo, L.A.A. Pertesson, M.R. Anderson e O. Inganas, Adv. Materials 10, 774 (1998).

[39] F. Thomazi, C.K. Saul, C.E.B. Marino, E. Burkarter e C.A. Dartora, Current Nanoscience 12, 611 (2016).

[40] F. Thomazi, M. Souza, C.K. Saul, G.A. Viana, F.C. Marques, R. Silvestre, M. Brehm, C.E.B. Marino, E. Burkarter e C.A. Dartora, Current Nanoscience 10, 877 (2014).

[41] B.R. Weinberger, M. Akhtar e S.C. Gau, Synthetic Metals 4, 187 (1982).

[42] C.W. Tang, Appl. Phys. Lett. 48, 183 (1986).

[43] R. Reshma Gopi e S. Sreejith, Renewable and Sustainable Energy Reviews 94, 1 (2018).

[44] I. Mathews, S.N. Kantareddy, T. Buonassisi e I. Marius Peters, Joule 3, 1415 (2019).

[45] R. Deng, N.L. Chang, Z. Ouyang e Chee Mun Chong, Renewable and Sustainable Energy Reviews 109, 532 (2019).

[46] P.G.V. Sampaio, M.O. Aguirre González, R.M. de Vasconcelos, M.A.T. dos Santos e J.P. Pinheiro Pereira, Renewable and Sustainable Energy Reviews 93, 215 (2018).

[47] P. Choudhary e R.K. Srivastava, Journal of Cleaner Production 227, 589 (2019).

[48] E.T. Jaynes e F.W. Cummings, Proc. IEEE 51, 89 (1963).

[49] F.W. Cummings, Phys. Rev. 140, A1051 (1965).

[50] F.W. Cummings, Journal of Physics B 46, 220202 (2013).

[51] J.J. Sakurai, Modern Quantum Mechanics (Addison Wesley, Boston, 1994).
[52] R. Eisberg e R. Resnick, Física Quântica (Elsevier Campus, Rio de Janeiro, 1979).

[53] J.D. Jackson, Classical Electrodynamics (John Wiley and Sons, Nova Jersey, 1998).

[54] K. Seeger, Semiconductor Physics (Springer, Berlim, 1997).

[55] S.M. Sze, Physics of semiconductor devices (Wiley, New York, 1981).

[56] J. Frenkel, Physical Review. 37, 17 (1931).

[57] W.Y. Liang, Physics Education 5, 226 (1970).

[58] S.M. Rezende, Materiais e dispositivos eletrônicos (Editora Livraria da Física, São Paulo, 2004).

[59] D.L. Staebler e C.R. Wronski, Applied Physics Letters 31, 292 (1977).

[60] A. Serpi, Physica Status Solidi A 133, K73 (1992).

[61] J.N. Heyman, J.D. Stein, Z.S. Kaminski, A.R. Banman, A.M. Massari e J.T. Robinson, Journal of Applied Physics 117, 015101 (2015).

[62] B. Náfrádi, Nature Communications 7, 13406 (2016).

[63] P. Würfel, The Physics of Solar Cells (Wiley-VCH, Weinheim, 2005).

[64] https://ieeexplore.ieee.org/document/347065/ metrics\#metrics

[65] I.F.L. Dias e M.A.T. da Silva, Polímeros Semicondutores (Editora Livraria da Física, São Paulo, 2012).

[66] S. Sharma, K.K. Jain, A. Sharma, Materials Sciences and Applications 6, 1145 (2015).

[67] A.M. Bagher, M.M.A. Vahid, M. Mohsen, American Journal of Optics and Photonics 3, 94 (2015).

[68] J. Jean, P.R. Brown, R.L. Jaffe, T. Buonassisi, V. Bulovic, Energy \& Environmental Science 8, 1200 (2015).

[69] M.Z. Iqbal, S.R. Ali e S. Khan, Solar Energy 181, 490 (2019).

[70] W. Shockley e H.J. Queisser, Journal of Applied Physics 32, 510 (1961)

[71] S. Yun, Y. Qin, A.R. Uhl, N. Vlachopoulos, M. Yin, D. Li, X. Han e A. Hagfeldt, Energy Environ. Sci. 11, 476 (2018).

[72] K.M. Coakley e M.D. McGehee, Chem. Mater. 16, 4533 (2004).

[73] B. O'Regan e M.A. Grätzel, Nature 353, 737 (1991).

[74] A. Fakharuddin, R. Jose, T.M. Brown e F. FabregatSantiago, J. Bisquert, Energy Environ. Sci. 7, 3952 (2014).

[75] H.J. Snaith, Nature Materials 17, 372 (2018).

[76] S. Ahmad, J.H. Yum, S. Xianxi, M. Grätzel, H.J. Butt e M.K. Nazeeruddin, Journal Of Materials Chemistry 20, 1654 (2010).

[77] K. Kakiage, Y. Aoyama, T. Yano, K. Oya, J. Fujisawa e M. Hanaya, Chemical Communications 51, 15894 (2015).

[78] J.S. Agnaldo, J.B.V. Bastos, J.C. Cressoni e G.M. Viswanathan, Revista Brasileira de Ensino de Física 28, 77 (2006).

[79] R. da Silva, A.J. Chiquito, M.G. de Souza e R.P. Macedo, Revista Brasileira de Ensino de Física 26, 379 (2004). 